\title{
GRADED RINGS OF RANK 2 SARKISOV LINKS
}

\author{
GAVIN BROWN AND FRANCESCO ZUCCONI
}

\begin{abstract}
We compute a class of Sarkisov links from Fano 3-folds embedded in weighted Grassmannians using explicit methods for describing graded rings associated to a variation of geometric invariant theory (GIT) quotient.
\end{abstract}

\section{$\S 1$. Introduction}

This paper describes a class of Sarkisov links from Fano 3-folds embedded in weighted Grassmannians $w$ Grass $(2,5)$. Altınok [Alt98] lists 69 families of such Fano 3-folds. These are listed in [BDK+] with the projection calculus of their K3 sections in [Bro07]. They are discussed from the point of view of weighted Grassmannians in [CR02]. The general member of each family lies in weighted projective space (WPS) as a codimension 3 quasi-smooth variety defined by the five maximal Pfaffians of a skew $5 \times 5$ matrix.

We make a detailed study of links from Type I centres on these Fano 3folds. (A Type I centre is a terminal cyclic quotient singularity $(1 / r)\left(n_{1}, n_{2}\right.$, $\left.n_{3}\right)$ on $X$ for which the weights $n_{1}, n_{2}, n_{3}$ of the $\mathbb{Z} / r \mathbb{Z}$ action are those of independent global variables (see Definition 3.1 below). The Kawamata blowup is then simply the weighted blowup with weights $n_{1}, n_{2}, n_{3}$.) Of Altınok's 69 families, 64 have a member with a Type I centre. Members of the remaining five families do not have a Type I centre (two of them have no projections at all, and the rest have more complicated projections that will need a new analysis starting with [Rei02] and generalisations of [Pap06]), and we do not study those cases here.

Received July 17, 2008. Accepted June 29, 2009.

2000 Mathematics Subject Classification. Primary 14J30; Secondary 14J45, 14E30, $14 \mathrm{E} 08$.

The first author was partially supported by Engineering and Physical Sciences Research Council grants GR/S03980/01 and EP/E000258/1 and by Ministero dell'istruzione, dell'Università e della Ricerca, Programmi di Ricerca di Rilevante Interesse Nazionale 2003: Spazi di moduli e teoria di Lie.

(C) 2010 by The Editorial Board of the Nagoya Mathematical Journal 
THEOREM 1.1. Let $X$ be a general member of one of the 64 families of codimension 3 Pfaffian Fano 3-folds admitting a Type I centre, and let $p \in X$ be a Type I centre. Suppose that the three quasi-linear equations defining the orbifold tangent space at $p$ have weights $a_{1} \leq a_{2} \leq a_{3}$. Then there is $a$ Sarkisov link that starts with the Kawamata blowup of $p$ followed by a flop:

$$
X \longleftarrow Y \rightarrow Y_{1}
$$

The link is completed in one of four ways:

$$
\begin{array}{ll}
a_{1}=a_{2}=a_{3}: & Y_{1}=Y^{\prime} \rightarrow \mathbb{P}^{2} \text { is a conic bundle; } \\
a_{1}<a_{2}=a_{3}: & Y_{1}=Y^{\prime} \rightarrow X^{\prime} \text { is a divisorial contraction to a line on a } \\
& \text { Fano } 3 \text {-fold } X^{\prime} ; \\
a_{1}=a_{2}<a_{3}: & Y_{1}-\rightarrow Y^{\prime} \rightarrow \mathbb{P}^{1} \text { is a Mori flip followed by a del Pezzo fibra- } \\
& \text { tion; } \\
a_{1}<a_{2}<a_{3}: & Y_{1}-\rightarrow Y^{\prime} \rightarrow X^{\prime} \text { is a Mori flip followed by a divisorial con- } \\
& \text { traction to a point on a Fano 3-fold } X^{\prime} .
\end{array}
$$

Theorem 1.1 is a concise statement of the result. Its proof, given in Sections 3.1 and 3.2, provides more detailed information about each of the four cases; this is given in Section 3.3, and it includes a list of conic bundles that occur and, in Table 1, a list of del Pezzo fibrations that occur. A great deal of additional intricate information about the links is gained by embedding them in toric links, and this is described in Sections 4 and 5.

Sarkisov links are a tool to study the birational rigidity (or lack thereof) of Fano 3-folds and Mori fibre spaces. In every link arising in Theorem 1.1, the 3 -fold at the end of the link (either $X^{\prime}$ or $Y^{\prime}$, depending on the link) is not isomorphic to the 3 -fold $X$ at the beginning. Thus we have the following.

Corollary 1.2. Let $X$ be a general member of one of the 64 families of codimension 3 Pfaffian Fano 3-folds that admit a Type I centre. Then $X$ is not birationally rigid.

\subsection{Explicit Sarkisov links in the literature}

Theorem 1.1 is a descendent of other explicit calculations in the literature. We describe some of these; we do not discuss work, such as that of Grinenko [Gri01] or Abramovich et al. [AKMW02], which is very relevant but proceeds by other methods.

For general weighted hypersurfaces $X \subset \mathbb{P}^{4}$, the full analysis of Sarkisov links from $X$ was completed in [CPR00]. It is shown there that all such links 
are birational involutions and therefore that a general Fano hypersurface is birationally rigid. In this context, Type I centres are those centres which lead to quadratic involutions (QI). Hypersurfaces also admit elliptic involutions (EI) that one might naturally define as Type $\mathrm{II}_{1}$ centres (see [Rei02] and [Pap06]).

In codimension 2, Corti and Mella [CM04] work out examples of links from $X \subset \mathbb{P}^{5}$ in detail and explain how to carry out calculations of links on any example. The main part of [CM04] is devoted to the 'exclusion' methods that show that particular Fano 3-folds have no other links. The following result is implicit in [CM04], and we phrase it as the prototype for Theorem 1.1.

Theorem 1.3 (Corti and Mella [CM04]). Let $X \subset \mathbb{P}^{5}$ be a codimension 2 Fano 3-fold, and let $p \in X$ be a Type I centre. Suppose that the equations defining the tangent space at $p$ have weights $a_{1} \leq a_{2}$. Then there is a Sarkisov link that starts with the Kawamata blowup of $p$ followed by a flop:

$$
X \leftarrow Y \rightarrow Y^{\prime}
$$

The link continues in one of two ways:

$$
\begin{array}{ll}
a_{1}=a_{2}: & Y^{\prime} \rightarrow \mathbb{P}^{1} \text { is a del Pezzo fibration; } \\
a_{1}<a_{2}: & Y^{\prime} \rightarrow X^{\prime} \text { is a divisorial contraction to a point on a Fano 3-fold } \\
& X^{\prime} .
\end{array}
$$

In the second case, the divisor contracts to a singular point $p^{\prime}$ on a Fano 3-fold $X^{\prime}$ and the index of $p^{\prime} \in X^{\prime}$ is $a_{2}-a_{1}$.

The results mentioned so far typically concern Fano 3-folds with rather small anticanonical systems. At the other end of the spectrum, Takagi [Tak06, Section 8.2], describes links from Fano 3-folds with large anticanonical systems to other Mori fibre spaces.

\subsection{Graded rings of blowups}

We construct links using variations of GIT. These methods were first discussed by Dolgachev and Hu [DH98], Reid [Rei92], and Thaddeus [Tha96]. They were used to construct Mori flips as toric hypersurfaces in [Bro99], and some of those flips appear in the links of Theorem 1.1. The more recent notion of Mori dream space of Hu and Keel [HK00] is very close to our needs here. The difference is that our computations are explicit: as discussed by Corti and Reid [CR00, introduction], we seek descriptions of every aspect 
of these links by equations or something equally concrete. We work directly with graded rings associated to the problem. One could define many rings, and during calculations there are two main considerations: we can work only with rings that are reasonably small, and we cannot always be certain that we have computed the whole ring under consideration. So, while the theory of [HK00] is comprehensive, we need other detailed information to make our calculations.

Let $X$ be a Fano 3-fold, and let $p \in X$. For an extremal contraction $f: Y \rightarrow X$ contracting a divisor $E \subset Y$ to a point $p \in X$, we follow Cox [Cox95], Hu and Keel [HK00], Kawakita [Kaw02], and others and describe the graded ring

$$
\mathcal{M}(f)=\bigoplus_{n \in \mathbb{Z}} \bigoplus_{m \geq 0} H^{0}\left(Y,-\left(m K_{Y}+n E\right)\right) \quad \text { (see Definition 5.2). }
$$

We use this graded ring to compute steps in a 2-ray game (see Section 2.1 below for details of 2-ray games).

THEOREM 1.4. Let $f$ be an extremal extraction from a point $p \in X$. If $\mathcal{M}(f)$ is finitely generated as a $\mathbb{C}$-algebra, then there is a 2-ray game $\Phi$ from $p \in X$ starting with $f$.

Of course, the finite generation condition on $\mathcal{M}$ is the essential point. To check it is tantamount to describing the link, and we do this for certain links from anticanonically embedded Fano 3 -folds in codimension 3 . We restate and prove this theorem as Theorem 5.4 below. The method of proof together with the explicit study of toric links in Section 4 gives us a way to analyse the steps in the Sarkisov links that appear.

This method describes other phenomena, such as Ryder's elliptic and K3 fibrations [Ryd06] and the 'bad' links of Corti et al. [CPR00]. The methods we describe are also used in [BCZ04] to compute links from Mori fibre spaces and in [CS05] to compute birational modifications.

Throughout this paper we work over the field $k=\mathbb{C}$.

\section{Acknowledgments}

This paper was initiated during the Higher Dimensional Geometry meeting of the Newton Institute, Cambridge, U.K., in 2002, and with visits of the first author to Udine, Italy, in 2003 and of the second author to the Department of Pure Mathematics and Mathematical Statistics, Cambridge, U.K., in 2002. We thank Professors A. Corti, M. Gross, M. Reid, and H. Takagi for 
their mathematical support during and since that time and two anonymous referees for suggesting numerous improvements to an earlier draft, pointing out, in particular, that the del Pezzo fibrations arising in Theorem 1.1 are not all standard.

\section{§2. Rank 2 links}

In the spirit of [CPR00], we consider explicit Fano 3-folds $X$ embedded in WPS $X \subset \mathbb{P}^{n}$ for small values of $n$ : we denote the weights of the WPS $\mathbb{P}^{n}$ by $\mathbb{P}^{n}\left(a_{0}, \ldots, a_{n}\right)$ when we know them, but even without this indication the symbol $\mathbb{P}^{n}$ always denotes some WPS. By definition, a Fano 3-fold has minimal Picard rank, $\rho_{X}=1$. There are well-known lists of such Fanos: Reid's famous 95 hypersurfaces, Iano-Fletcher's [IF00] 85 families in codimension 2, and Altınok's [Alt98] lists of 70 and 142 Fanos in codimensions 3 and 4. These lists, and others in higher codimension, are in $[\mathrm{BDK}+]$. We sometimes assume that $X$ is quasi smooth, that is, that the weighted affine cone on $X$ is singular only at the origin. Quasi-smooth varieties in WPS have only quotient singularities.

Birational links and the Sarkisov program are already well discussed (see e.g., [Cor00]), so we are brief. We recall that a 3 -fold $X$ is $\mathbb{Q}$-factorial if every Weil divisor on $X$ admits some multiple that is a Cartier divisor. When this condition fails for a divisor $D$, there is a projective morphism $Y \rightarrow X$ that is an isomorphism in codimension 1 so that the strict transform of $D$ is a (relatively ample) Cartier divisor (see [Kaw88, Lemma 3.1] for a more detailed explanation).

\subsection{The 2-ray game and rank 2 links}

The key observation is that if a projective and $\mathbb{Q}$-factorial variety $Y$ has Picard rank $\rho(Y)=2$, then up to isomorphism it admits at most two projective morphisms, each of relative Picard rank 1. There are two instant sources of such varieties: fibrations $Y \rightarrow S$ and blowups $Y \rightarrow X$, where in each case the base is assumed $\mathbb{Q}$-factorial with $\rho=1$ and the map is extremal in the sense that $\rho(Y / S)=1$ or $\rho(Y / X)=1$. Each of these descriptions of $Y$ accounts for one of the two possible extremal morphisms that could exist from $Y$. If $Y$ admits a second morphism, $Y \rightarrow Z$, say, then one of two things may happen. If $Z$ is $\mathbb{Q}$-factorial, then the game is over. On the other hand, if $Z$ is not $\mathbb{Q}$-factorial, then the morphism is necessarily small and one looks to make a flip

$$
Y \rightarrow Z \leftarrow Y_{1}
$$


meaning that the rational map $Y \rightarrow Y_{1}$ is an isomorphism in codimension 1 but is not an isomorphism, and that the flipped variety $Y_{1}$ is again $\mathbb{Q}$-factorial with $\rho=2$. And so the 2-ray game proceeds: we switch attention to $Y_{1}$, look for its second morphism $Y_{1} \rightarrow Z_{2}$-again, one of the two possible morphisms from $Y_{1}$ is already accounted for-and terminate or flip as required. From either of our starting configurations, there is never a choice to be made as long as the necessary morphisms and flips exist. However, the game can break down by encountering either a $Y_{i}$ with only one morphism or a flipping contraction $Y_{i} \rightarrow Z_{i+1}$ that does not flip.

The expression 2-ray game refers to this procedure, whether or not it breaks down. Successful games are called 2-ray links.

Definition 2.1. A 2-ray link is a diagram

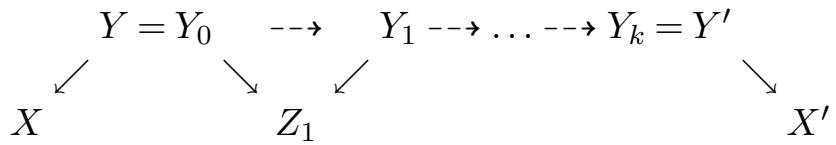

of projective varieties and projective morphisms, in which each $Y_{i}$ is $\mathbb{Q}$ factorial with $\rho\left(Y_{i}\right)=2, X$ and $X^{\prime}$ are $\mathbb{Q}$-factorial with $\rho=1$, and each $Z_{i}$ has $\rho\left(Z_{i}\right)=1$ but is not $\mathbb{Q}$-factorial. Moreover, the rational maps $Y_{i} \rightarrow Y_{i+1}$ are isomorphisms in codimension 1 but are not isomorphisms.

\subsection{The Sarkisov program}

Now we discuss the category in which we play a 2-ray game. Many are appropriate, but we consider the Mori 'category' of projective, $\mathbb{Q}$-factorial, terminal 3-folds and extremal morphisms. As is familiar, such 3-folds occur naturally in the minimal model program in three dimensions, even if one starts with nonsingular 3-folds (see [KM98] for a general introduction). We refer to [Rei87] for the definition of terminal singularities, since we do not use it directly.

Definition 2.2. (1) A morphism $f: Y \rightarrow X$ is extremal if $f_{*} \mathcal{O}_{Y}=\mathcal{O}_{X}$ and $\rho(Y / X)=1$.

(2) An extremal contraction is an extremal (projective) morphism $f: Y \rightarrow$ $X$ between projective, $\mathbb{Q}$-factorial varieties such that $-K_{Y}$ is relatively ample for $f$. We say that $f$ is of divisorial type if $\operatorname{dim} X=\operatorname{dim} Y$ and that $f$ is of fibre type if $\operatorname{dim} X<\operatorname{dim} Y$.

(3) A 2-ray link - with notation as in (2.1) of Definition 2.1 - is said to take place in the Mori category if 
(i) each $Y_{i}$ is a 3 -fold with terminal singularities;

(ii) $\operatorname{dim} X \leq 3$, and if $\operatorname{dim} X=3$, then $X$ has terminal singularities, and the same conditions for $X^{\prime}$;

(iii) $Y \rightarrow X$ and $Y^{\prime} \rightarrow X^{\prime}$ are extremal contractions; and

(iv) all morphisms $Y_{i} \rightarrow Z_{j}$ (for $j=i$ or $i+1$ and $Z_{j} \neq X, X^{\prime}$ ) are extremal and small.

If $f$ is an extremal contraction of divisorial type, then necessarily $f$ contracts an irreducible divisor $E \subset Y$ to a subvariety of codimension at least 2 in $X$.

Definition 2.3. A Mori fibre space (MFS) is an extremal contraction $V \rightarrow S$ of fibre type with $\operatorname{dim} V=3$ in which $V$ has terminal singularities.

There are three cases of MFS $V \rightarrow S$, defined as follows:

if $\operatorname{dim} S=0$, then $V$ is a Fano 3-fold;

if $\operatorname{dim} S=1$, then $V$ is a del Pezzo fibration;

if $\operatorname{dim} S=2$, then $V$ is a conic bundle.

Definition 2.4. A Sarkisov link between two MFS $V \rightarrow S$ and $V^{\prime} \rightarrow S^{\prime}$ is a 2-ray link that takes place in the Mori category in which both $V$ and $V^{\prime}$ appear.

Of course, in Definition 2.4 the two MFS necessarily appear at the two ends of the 2-ray link. But we can be more specific. There are four configurations - named Types I-IV in [Cor00, Section 2.2] in which $V$ and $V^{\prime}$ can appear in a 2-ray link, depending on the dimensions of $S$ and $S^{\prime}$ :

Type I, $\quad V$ and $V^{\prime}$ are Fano 3 -folds;

Type II, $\quad V$ is a Fano 3 -fold and $V^{\prime} \rightarrow S^{\prime}$ is of fibre type;

Type III, $\quad V \rightarrow S$ is of fibre type and $V^{\prime}$ is a Fano 3 -fold;

Type IV, $\quad V \rightarrow S$ and $V^{\prime} \rightarrow S^{\prime}$ are of fibre type.

There is only one way that each type can fit into a link: comparing with the link (2.1) of Definition 2.1, for Type I, $V=X$ and $V^{\prime}=X^{\prime}$; for Type II, $V=X$ and $\left(V^{\prime} \rightarrow S^{\prime}\right)=\left(Y^{\prime} \rightarrow X^{\prime}\right)$; and so on. The links in Theorem 1.1 all start with $(V \rightarrow S)$ being a Fano 3 -fold $(X \rightarrow$ pt.), and the first step being a blowup $Y \rightarrow X$ of a Fano 3 -fold, so they are all of Type I or II. 
We have given these definitions over $\operatorname{Spec}(k)$. They also make sense over arbitrary base scheme $U$, with all notions replaced by their relative counterparts: $\rho(Y)=2$ is replaced by $\rho(Y / U)=2$, and so on. This is done carefully in [Cor00].

\section{$\S 3$. General Fano 3-folds in codimension 3}

\subsection{The equations of Fano 3-folds}

3.1.1. Weighted Grassmannians and Pfaffian equations. We consider explicit Fano 3 -folds $X \subset \mathbb{P}^{6}=\mathbb{P}^{6}\left(a_{0}, \ldots, a_{6}\right)$ other than the standard complete intersection $X_{2,2,2} \subset \mathbb{P}^{6}$. There are 69 families of such Fano 3-folds, and they are listed in $[\mathrm{BDK}+]$ with their degrees, baskets, and Hilbert numerators (see [ABR02, Section 4.6] or [Bro07, Section 2.1] in the context of K3 surfaces, for an account of these notions).

A general member of each family is quasi smooth and has singularities equal to those of the basket. It has equations that are the five maximal Pfaffians of a skew $5 \times 5$ matrix of homogeneous forms, and it is easy to construct a general member by writing down such a matrix; this matrix is also the first syzygy matrix of the equations, so the degrees of forms appearing are determined by the Hilbert numerator. When focusing on a particular singular point of such a Fano, we can usually make coordinate changes to fix a large proportion of the free parameters since this does not change the loci where the matrix drops rank. We illustrate this with Family No. 10 of $[\mathrm{BDK}+]$.

Family No. 10. Here $X \subset \mathbb{P}^{6}(1,1,1,2,2,2,3$ with degree $=13 / 6$ and basket $=\{3 \times 1 / 2(1,1,1), 1 / 3(1,1,2)\})$. This example has Hilbert numerator $1-3 t^{4}-2 t^{5}+2 t^{6}+3 t^{7}-t^{11}$, so we construct the five Pfaffian equations to include three of degree 4 and two of degree 5 . Let $x, x_{1}, x_{2}, y, y_{1}, y_{2}, z$ be the weighted homogeneous coordinates of $\mathbb{P}^{6}$, in that order. We consider each type of singularity in turn as a centre.

We move a singularity of type $1 / 2(1,1,1)$ at the coordinate point $P_{y}$ by a change of coordinates. To ensure $P_{y} \in X$ is quasi smooth, the equations near $P_{y}$ must eliminate the variables $y_{1}, y_{2}$ and one other. This is easily arranged by writing the syzygy matrix as

$$
\left(\begin{array}{cccc}
y & A_{3} & B_{3} & C_{2} \\
& D_{2} & E_{2} & F_{1} \\
& & z & y_{2} \\
& & & y_{1}
\end{array}\right)
$$


where $A, \ldots, F$ are general forms of the degrees indicated. By row and column operations on the matrix, we assume further that $y$ does not appear in $A, \ldots, E$ and that $F_{1}=x$. Apart from being quasi smooth, the only appeal to generality is that the $3-4$ entry of the syzygy matrix is $z$ rather than $x y$. (In [CPR00], this kind of assumption that a particular monomial appears in the equations is called starred monomial. It is needed for the vanishing calculation of Lemma 3.6 below, and omitting the starred monomial will typically change the link.)

The link from the $1 / 3(1,1,2)$ point can be best computed by presenting the equations of a general $X$ by the syzygy matrix

$$
\left(\begin{array}{cccc}
z & A_{3}^{\prime} & B_{2}^{\prime} & C_{2}^{\prime} \\
& D_{3}^{\prime} & E_{2}^{\prime} & F_{2}^{\prime} \\
& & y_{1} & y \\
& & & x
\end{array}\right)
$$

for general forms $A^{\prime}, \ldots, F^{\prime}$. It is possible to write the syzygy matrix so that both of the links above can be 'seen' in its format, but we do not attempt to do this.

3.1.2. Type $I$ singularities. If $X \subset \mathbb{P}^{n}$ is quasi smooth at $p \in X$, then there must be $n-3$ polynomials in the ideal of $\mathcal{O}_{X, p}$ that vanish and are independently linear near $p$. If, as is typical, $p=p_{w}$ is at a coordinate point of $\mathbb{P}^{n}$, then the ideal of $X$ must contain $n-3$ equations of the form

$$
w^{k} \ell=w^{k-1} m_{k-1}+\cdots+m_{0},
$$

with $\ell$ containing at least one term that is a linear variable - that is, $\ell$ is a quasi-linear form - and polynomials $m_{i}$ in the other variables. The $n-3$ homogeneous quasi-linear polynomials like $\ell$ are called the tangent polynomials at $p$.

Definition 3.1 ([ABR02, Section 5.5]). Let $X \subset \mathbb{P}^{n}$ be a Fano 3-fold, and let $p \in X$ be a quasi-smooth point of $X$; in particular, $p \in X$ is a quotient singularity of type $(1 / r)(1, a, r-a)$ for some coprime $0<a<r$. Then $p \in X$ is a Type $I$ point if and only if $p$ lies at one of the coordinate points $p=p_{w}$ of $\mathbb{P}^{n}$ and there are coordinate functions $x, y, z$ on $\mathbb{P}^{n}$ that vanish at $p$, have weights $1, a, r-a$, respectively, and are independent in $m_{p} / m_{p}^{2}$ modulo the tangent polynomials.

A Type I point $p \in X$ is a Type I centre if and only if

$$
-K_{X}^{3}>1 /(r a(r-a))
$$


This definition is perfect for our calculations, although it would be slightly awkward to use more generally: the term centre usually applies to a component of the base locus of a linear system which is determined by a birational map, and this definition is anticipating such a map.

We illustrate the definition with a nonexample. The Fano 3-fold

$$
X_{66}:\left(x^{66}+x y^{13}+z^{11}+t^{3}-u^{2}=0\right) \subset \mathbb{P}(1,5,6,22,33)
$$

(with variables $x, y, z, t, u$ in that order) is quasi smooth. It contains the point $p=(0,0,0,1,1)$ as a quotient singularity of type $(1 / 11)(1,5,6)$. But $p \in X$ is not a Type I point according to the definition, because it does not (and cannot even after coordinate changes) lie at a coordinate point.

Proposition 3.2. Let $X \subset \mathbb{P}^{6}$ be a general member of the 64 codimension 3 families of Fano 3-folds that admit a Type I centre $p \in X$. We may choose coordinates so that $p$ lies at a coordinate point $p_{t} \in \mathbb{P}^{6}$. Then the syzygy matrix of $X \subset \mathbb{P}^{6}$ can be written as

$$
\left(\begin{array}{cccc}
t & A & B & C \\
& D & E & F \\
& & y_{3} & y_{2} \\
& & & y_{1}
\end{array}\right)
$$

where $y_{1}, y_{2}, y_{3}$ are the tangent polynomials at $p$ and $A, \ldots, F$ are forms (of degree determined by the Hilbert numerator of $X$ ) in all the variables. Without loss of generality, $y_{1}, y_{2}, y_{3}$ are three distinct coordinate functions on $\mathbb{P}^{6}$.

Proof. All claims in this proposition come from direct observation of the list of codimension 3 Fanos. The form of the syzygy matrix follows from the definition of Type I point after one has checked that the equations are of the form $t y_{i}=\cdots$ rather than $t^{2} y_{i}=\cdots$ or even higher powers of $t$ in the tangent monomial.

Notation 3.3. Let $p \in X$ be a Type I centre on a Fano as in Proposition 3.2 , and suppose that $p \in X$ is a singularity of type $(1 / r)(a, b, c)$. We choose coordinates so that

- $X \subset \mathbb{P}^{6}\left(a, b, c, r, a_{1}, a_{2}, a_{3}\right)$ with coordinates $x, y, z, t, y_{1}, y_{2}, y_{3}$, in that order;

- $p=p_{t} \in X$ is the $t$-coordinate point;

- $x, y, z$ are the eigencoordinates of the $\mathbb{Z} / r$ stabiliser at $p$; 
- $y_{1}, y_{2}, y_{3}$ are the tangent polynomials at $p$; and

- the weights of the tangent polynomials satisfy $a_{1} \leq a_{2} \leq a_{3}$.

The variables $x, y, z$ are also called the polarising variables at $p$.

Corollary 3.4 (of Proposition 3.2). Using Notation 3.3, let $\Pi$ be the locus $t=y_{1}=y_{2}=y_{3}=0$ in $\mathbb{P}^{6}$. Then $\Pi \cong \mathbb{P}^{2}(a, b, c)$ and $X \cap \Pi$ is the locus defined by the three $2 \times 2$ minors

$$
\left(\bigwedge^{2}\left(\begin{array}{lll}
A & B & C \\
D & E & F
\end{array}\right)=0\right) \subset \Pi=\mathbb{P}^{2}(a, b, c),
$$

which is a finite reduced set of nonsingular points of $\mathbb{P}^{2}(a, b, c)$.

In fact, setting $d=\operatorname{deg} A-\operatorname{deg} D$ (which equals $\operatorname{deg} B-\operatorname{deg} E$ and $\operatorname{deg} C-$ $\operatorname{deg} F)$ and

$$
d_{i}=\frac{1}{2}\left((-1)^{i} d+\sum_{j=1}^{3}\left(a_{j}+r\right)\right) \quad \text { for } i=1,2,
$$

the number of points of $X \cap \Pi$ is $N=\sum_{i \neq j}\left(a_{i}+r\right)\left(a_{j}+r\right)-d_{1} d_{2}$.

Proof. The finiteness of $X \cap \Pi$ is automatic since otherwise the displayed minors would define a divisor in $X$ contradicting $\rho_{X}=1$. The extra claim is that the degrees of these minors are sufficiently high and divisible in each case such that they determine free linear systems on $\mathbb{P}^{2}(a, b, c)$, from which it follows that, for general $X$, these minors have common solutions only away from the toric strata of $\mathbb{P}^{2}(a, b, c)$. This is checked for each of the 64 cases in turn.

The counting argument is standard use of the Hilbert-Burch theorem. If $Z=X \cap \Pi$, then there is an exact sequence of $\mathcal{O}=\mathcal{O}_{\Pi \text {-modules }}$

$$
0 \rightarrow \mathcal{O}\left(-d_{1}\right) \oplus \mathcal{O}\left(-d_{2}\right) \rightarrow \bigoplus_{i=1}^{3} \mathcal{O}\left(-\left(a_{i}+r\right)\right) \rightarrow \mathcal{O} \rightarrow \mathcal{O}_{Z} \rightarrow 0
$$

(for suitable $d_{1}, d_{2}$ ), where the two nontrivial maps are given by the matrix

$$
M=\left(\begin{array}{lll}
A & B & C \\
D & E & F
\end{array}\right)
$$

and the vector of its (signed) $2 \times 2$ minors, respectively. We compute chern classes to conclude. The equality of $c_{1}$ for the third and fourth terms gives the formula for $d_{i}$; that is, comparison of degrees in the exact sequence. Then computing the Hilbert polynomial of $Z$, or equivalently $c_{2}$, gives the required number. 


\subsection{The proof of Theorem 1.1}

Let $Y \longrightarrow X$ be the Kawamata blowup of $p$ with exceptional divisor $E \subset Y$; note that $\rho_{Y}=2$. Define $F_{i} \subset Y$ to be the birational transform on $Y$ of $\left(y_{i}=0\right) \subset X$.

Lemma 3.5. The intersection $\Gamma=F_{1} \cap F_{2} \cap F_{3}$ is a reduced union of disjoint rational curves $\Gamma=\cup \Gamma_{i}$. For each $i$,

$$
E \Gamma_{i}=1 \quad \text { and } \quad A \Gamma_{i}=1 / r,
$$

so $B \Gamma_{i}=0$ and $\Gamma_{i} \stackrel{\text { num }}{\sim} \Gamma_{j}$ for all $i, j$. (The number of components $\Gamma_{i} \subset \Gamma$ is the number of points, $N$, computed in Corollary 3.4.)

Proof. By Corollary 3.4, $\Gamma_{X}=\left(y_{1}=y_{2}=y_{3}=0\right) \subset X$ is the cone on a finite set of reduced points with vertex at $p$-in particular, its components are irreducible rational curves. The Kawamata blowup is the blowup of the ideal at $p$ generated by the polarising variables, so $E \subset Y$, the preimage of $p$, is isomorphic to $\mathbb{P}(a, b, c)$, and the birational transform of $\Gamma_{X}$ intersects $E$ in the same Hilbert-Burch locus as $X \cap \Pi$.

Thus $E \Gamma_{i}=1$, and $A \Gamma_{i}=1 / r$ because $r A \cap \Gamma$ computed on $Y$ is the same as $\Pi \cap \Gamma_{X}$. The numerical equivalence of $\Gamma_{i}$ follows since $\rho_{Y}=2$ implies that the pair $A, E$ spans $\overline{\mathrm{NE}}^{1}(Y)$.

We denote $A=-K_{X}$ and $B=-K_{Y}$, and we record that on $Y$

$$
B=A-(1 / r) E \quad \text { and } \quad E^{3}=r^{2} /(a b c),
$$

so Lemma 3.5 shows that $B \Gamma_{i}=0$.

Any function $f$ of weight $k$ on $X$ with $f(p)=0$ vanishes to order at least $k / r$ on $E$ when pulled back to $Y$. For the polarising variables $x, y, z$, as functions on $Y$ we have

$$
x \in \mathcal{O}_{Y}\left(a A-\frac{a}{r} E\right), \quad y \in \mathcal{O}_{Y}\left(b A-\frac{b}{r} E\right), \quad z \in \mathcal{O}_{Y}\left(c A-\frac{c}{r} E\right)
$$

(see, e.g., [CPR00, Proposition 3.4.6]). By the next lemma, the tangent polynomials $y_{i}$ vanish on $E$ exactly once more than generic functions of their degree.

Lemma 3.6. For each $i=1,2,3$, we have

$$
F_{i} \sim_{\mathbb{Q}} a_{i} A-\left(\left(a_{i}+r\right) / r\right) E=a_{i} B-E,
$$

where $\sim_{\mathbb{Q}}$ denotes $\mathbb{Q}$-linear equivalence (and so a fortiori numerical equivalence). 
Proof. By Proposition 3.2, $t$ does not vanish near $p \in X$ and

$$
y_{i}=\frac{i \text { th minor }}{t} \in \mathcal{O}\left(a_{i} A\right)
$$

Pulling this back to $Y$, the minor has degree $a_{i}+r$, so it vanishes to order at least $\left(a_{i}+r\right) / r$ along $E$. But in each case, the minor includes a nontrivial monomial in the polarising variables $x, y, z$-by generality of $X$ in its family - so that by (3.1) the vanishing is exactly $\left(a_{i}+r\right) / r$ along $E$ as claimed.

According to this lemma, we can sketch in $N^{1}(Y) \cong \mathbb{R}^{2}$ the rays through the various divisors as follows (noting that rays through the $F_{i}$ may coincide):

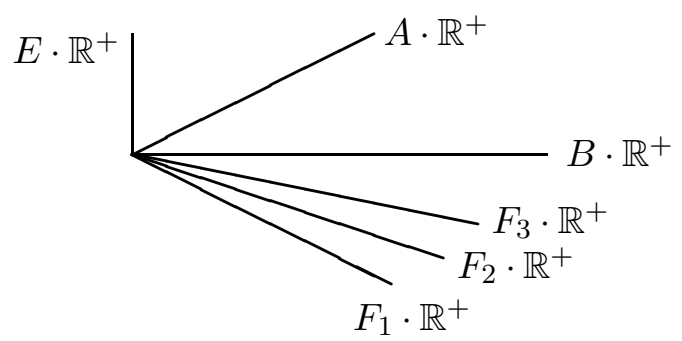

Corollary 3.7. The divisor $B$ is nef on $Y$, and it supports an extremal ray of $\overline{\mathrm{NE}}(Y)$; in the notation of Lemma 3.5, that ray is $\mathbb{R}_{+}[\Gamma]$, and it is a flopping ray.

Proof. Lemma 3.6 implies that $\left(a_{i}+r\right) B=F_{i}+r A$, so if $B \gamma<0$ then $\gamma \subset \cap F_{i}$. So $B$ is nef since each $B \Gamma_{i}=0$, and moreover it supports the ray $\mathbb{R}_{+}[\Gamma]$.

Let $Y \longrightarrow Z$ be the contraction of the ray $\mathbb{R}_{+}[\Gamma]$. Then $Z$ has Picard rank 1 and $B$ is the ample generator, so in particular $Z$ is a (non- $\mathbb{Q}$-factorial) Fano 3-fold.

Lemma 3.8. Let $Z \longleftarrow Y_{1}$ be the flop of $Y \longrightarrow Z$. Then on $Y_{1}$,

(a) the intersection $F_{1} \cap F_{2} \cap F_{3}$ is empty.

(b) $F_{3}$ is nef.

(c) $F_{1} \cap F_{2}$ is a nonempty union of curves, and $F_{3} C=0$ for every curve $C \subset F_{1} \cap F_{2}$.

(d) The second extremal ray of the Mori cone $\overline{\mathrm{NE}}\left(Y_{1}\right)$ equals $\mathbb{R}_{+}[C]$, where $C$ is any reduced and irreducible component of $F_{1} \cap F_{2}$. This ray is supported by $F_{3}$, and it is contracted by the extremal morphism $\varphi_{F_{3}}$ given by a positive multiple of $F_{3}$. 
Proof. (a) The flop $Z \leftarrow Y_{1}$ restricted to $E \cong \mathbb{P}(a, b, c)$ is the blowup of the locus of points $\left(y_{1}=y_{2}=y_{3}=0\right) \subset E \subset Z$. This is the same locus as computed in Corollary 3.4 since the $y_{i}$ are equal to the minors on $E$. To make the flop is to blow up this locus, and by construction, the generators of the blowup ideal have no common solutions on the blowup.

(b) If on $Y_{1}$ a curve $\gamma$ has $F_{3} \gamma<0$, then also $F_{i} \gamma<0$ for $i=2,3$, since $a_{3} \geq a_{i}$, so $\gamma \subset F_{1} \cap F_{2} \cap F_{3}$. But there are no such curves, so $F_{3}$ is nef on $Y_{1}$.

(c) The intersection of any pair $F_{i} \cap F_{j}$ does not contain a surface (since $Y \rightarrow Y_{1}$ is an isomorphism in codimension 1), so it is at most onedimensional. We compute $F_{1} \cap F_{2}$ on $X$ - the map to $Y_{1}$ is a blowup of $p \in X$ followed by a flop, so the result follows. Setting $y_{1}=y_{2}=0$ in the equations of $X$ cuts out the flopping curves $\Gamma_{X}$ together with the distinct locus

$$
\left(C=F=y_{3} t-A E+B D=0\right) \subset \mathbb{P}^{4}\left(r, a, b, c, a_{3}\right) .
$$

This latter locus is not empty and is at least one-dimensional. Its birational transform on $Y_{1}$ is the locus $F_{1} \cap F_{2}$ in question, and its intersection with $F_{3}$ is trivial by (a).

(d) Follows immediately from (a)-(c).

The continuation of the link from $Y_{1}$ depends on strictness of the inequalities $a_{1} \leq a_{2} \leq a_{3}$.

Proposition 3.9. Suppose that $a_{2}=a_{3}$. Then the contraction of the extremal ray by $\varphi_{F_{3}}: Y_{1} \rightarrow X^{\prime}$ determines a morphism to a $\mathbb{Q}$-factorial variety $X^{\prime}$. There are two subcases:

- if $a_{1}=a_{2}$, then $\operatorname{dim} X^{\prime}=2$ and $\varphi_{F_{3}}$ is a conic bundle;

- if $a_{1}<a_{2}$, then $\operatorname{dim} X^{\prime}=3$ and $\varphi_{F_{3}}$ is a divisorial contraction that contracts an irreducible divisor to a line in $X^{\prime}$.

Proof. By assumption, $F_{2} \sim F_{3}$, so by Lemma 3.8(a) the restriction of $F_{3}$ to $F_{1}$ contains a free pencil on $F_{1}$, and so it is in fact a multiple of a free pencil. In particular, there are effective curves $\gamma$ with $F_{3} \gamma=0$. Since $F_{3}$ is nef, any such curve generates an extremal ray, so the map $\varphi_{F_{3}}$ contracts $F_{1}$ to a line.

Now the classification of extremal contractions completes the proof. First, $\varphi_{F_{3}}$ is not small, so its image is $\mathbb{Q}$-factorial. If $a_{1}=a_{2}$, then $\varphi_{F_{3}}$ also contracts each of $F_{2}$ and $F_{3}$ to a line, so $\varphi_{F_{3}}$ can only be a conic bundle. If 
$a_{1}<a_{2}$, then on $Y_{1}$

$$
F_{3}^{3}=F_{3} F_{2}\left(F_{1}+\left(a_{3}-a_{1}\right) B\right)=\left(a_{3}-a_{1}\right) B F_{3} F_{2}>0
$$

since $F_{1} F_{2}=0$ on $Y_{2}$, where the final strict inequality holds because $F_{2} \cap F_{3}$ contains nonflopping curves. Thus the image of $\varphi_{F_{3}}$ is a 3 -fold, and the only possibility is that $\varphi_{F_{3}}$ is a divisorial contraction to a line.

Proposition 3.10. Suppose that $a_{2}<a_{3}$. Then the contraction of the extremal ray by $\varphi_{F_{3}}: Y_{1} \rightarrow Z_{2}$ is a flipping contraction and the flip $Z_{2} \leftarrow Y_{2}$ exists.

The second extremal ray of the Mori cone $\overline{\mathrm{NE}}^{1}\left(Y_{2}\right)$ is supported by the divisor $F_{2}$, and the contraction of this extremal ray $\varphi_{F_{2}}: Y_{2} \rightarrow X^{\prime}$ determines a morphism to a $\mathbb{Q}$-factorial variety $X^{\prime}$. There are two subcases:

- if $a_{1}=a_{2}$, then $\operatorname{dim} X^{\prime}=1$ and $\varphi_{F_{2}}$ is a del Pezzo fibration;

- if $a_{1}<a_{2}$, then $\operatorname{dim} X^{\prime}=3$ and $\varphi_{F_{2}}$ is a divisorial contraction that contracts an irreducible divisor to a point in $X^{\prime}$.

Proof. Since $a_{2}<a_{3}$, any curve $\gamma$ with $F_{3} \gamma=0$ has $F_{2} \gamma<0$ and $F_{1} \gamma<0$ and so lies in $F_{1} \cap F_{2}$. There are only finitely many such curves, so the extremal neighbourhood supported by $F_{3}$ is isolated; it is flipping because $B$ is nef on $Y_{1}$ and so is strictly positive on the ray.

The flip exists because the exceptional locus is cut out by two divisors and Mori's easy flip theorem ([Kol92, Theorem 20.11]) applies. Furthermore, by Mori's theorem, the intersection $F_{1} \cap F_{2}$ is empty on the flipped variety $Y_{2}$. (Of course, because it resolves the 'weighted pencil' $F_{1}: F_{2}$, Mori's theorem requires the two divisors to be proportional in $\operatorname{Pic}\left(Y_{1}\right)$, whereas $F_{1}$ and $F_{2}$ may not be. But they are proportional in a neighbourhood of the flipping locus. For instance, if we set $F_{1}^{\prime}=\left(a_{3}-a_{2}\right) F_{1}+\left(a_{2}-a_{1}\right) F_{3}$, which is linearly equivalent to $\left(a_{3}-a_{1}\right) F_{2}$, then $F_{1}^{\prime} \cap F_{2}$ equals $F_{1} \cap F_{2}$ set-theoretically away from $F_{3}$ because the flop cleared that triple intersection. Thus on $Y_{2}$ we have that $F_{1} \cap F_{2}=\emptyset$ off $F_{3}$, but again, because of the flop, this is also true on $F_{3}$.)

Let $Y_{2}$ be the flipped variety. As for $F_{3}$ in Lemma 3.8, $F_{2}$ is nef. In fact, $F_{2}$ supports the second extremal ray on $Y_{2}$, because it is trivial on any curve contained in $F_{1}$. Moreover, the extremal contraction $\varphi_{F_{2}}$ contracts $F_{1}$ and so is not small. Once again, the classification of extremal contractions finishes the proof. First, the image of $\varphi_{F_{2}}$ is $\mathbb{Q}$-factorial. If $a_{1}=a_{2}$, then $\varphi_{F_{2}}$ contracts any surface in the pencil $\left|F_{1}\right|$ to a point and so must be a del 
Pezzo fibration. If $a_{1}<a_{2}$, then

$$
F_{2}^{3}=F_{2}\left(F_{1}+\left(a_{2}-a_{1}\right) B\right)^{2}=\left(a_{2}-a_{1}\right)^{2} F_{2} B^{2}>0
$$

the positivity holding because $B$ is ample. Thus the image of $\varphi_{F_{2}}$ is a 3 -fold and the only possibility is that $\varphi_{F_{2}}$ is a divisorial contraction to a point.

\subsection{Numerical properties of the end of the link}

The calculations in the proof of Theorem 1.1 can also be used to compute detailed information about the varieties at the end of the link in each of the four cases. We use the notation of Theorem 1.1 throughout.

Proposition 3.11 (Divisorial contraction to a point). If $a_{1}<a_{2}<a_{3}$, then the image of the contracted divisor is a singular point $p^{\prime}$ on a Fano 3-fold $X^{\prime}$ and the index of $p^{\prime} \in X^{\prime}$ is an integer multiple of $a_{2}-a_{1}$.

Proof. The map $\varphi: Y^{\prime} \rightarrow X^{\prime}$ is given by (multiples of) the linear system of the divisor $F_{2}$, and it contracts the divisor $F_{1}$ to a point $q \in X^{\prime}$. Moreover, $X^{\prime}$ is a Fano 3 -fold, so denoting $A^{\prime}=-K_{X^{\prime}}$, we have

$$
\varphi^{*}\left(A^{\prime}\right)=B+\frac{a^{\prime}}{r^{\prime}} F_{1},
$$

where $r^{\prime}$ is the index of $q \in X^{\prime}$ and $a^{\prime}$ is some positive integer. Since $\rho_{X^{\prime}}=1$, the divisor $\varphi^{*}\left(A^{\prime}\right)$ must be some positive multiple, possibly rational, of $F_{2}$, say, $\varphi^{*}\left(A^{\prime}\right)=\lambda F_{2}$. So since $F_{2}=F_{1}+\left(a_{2}-a_{1}\right) B$ on $Y^{\prime}$, then

$$
\lambda F_{1}+\lambda\left(a_{2}-a_{1}\right) B=B+\frac{a^{\prime}}{r^{\prime}} F_{1} .
$$

But $B$ and $F_{1}$ are linearly independent in $\operatorname{Pic}\left(Y^{\prime}\right)$, so $r^{\prime}=a^{\prime}\left(a_{2}-a_{1}\right)$ is a positive integral multiple of $a_{2}-a_{1}$.

Notice that, in the notation of the proof, if $F_{1} \subset Y^{\prime}$ is Cartier, then the index is exactly $a_{2}-a_{1}$ : in that case, we can use an argument of Kawamata [Kaw96] to show that $a^{\prime}=1$. Let $V \stackrel{g}{\rightarrow} Y^{\prime} \rightarrow X^{\prime}$ be a resolution of singularities, with composition denoted $f$. If $a^{\prime} \neq 1$, then there is a divisor $G \subset V$ with discrepancy $1 / r^{\prime}$ over $X^{\prime}$. But then, away from all exceptional divisors other than $G$ and $F_{1}$, we have $f^{*}\left(-K_{X^{\prime}}\right)=-K_{V}+\left(a^{\prime} / r^{\prime}\right) F_{1}+\left(1 / r^{\prime}\right) G$ while also

$$
f^{*}\left(-K_{X^{\prime}}\right)=g^{*}\left(-K_{Y^{\prime}}+\left(a^{\prime} / r^{\prime}\right) F_{1}\right)=g^{*}\left(-K_{Y^{\prime}}\right)+\left(a^{\prime} / r^{\prime}\right) F_{1}+m G
$$


where $m \geq a^{\prime} / r^{\prime}$. Equating these two expressions shows that $Y^{\prime}$ does not have terminal singularities, which is not true. Kawakita's analysis of divisorial contractions (see [Kaw05]) distinguishes between cases when $F_{1}$ is Cartier or not, but we have not carried out the additional calculations to see which cases occur.

Proposition 3.12 (Conic bundle). If $a_{1}=a_{2}=a_{3}$, then the conic bundle $Y^{\prime} \rightarrow \mathbb{P}^{2}$ has discriminant $\Delta \subset \mathbb{P}^{2}$ of degree

$$
\operatorname{deg} \Delta=12-a_{1} \operatorname{deg}(X)+\left(\frac{a_{1}+r}{r}\right)\left(\frac{1}{a(r-a)}\right) .
$$

Proof. We know that $\left(-K_{F_{1}}\right)^{2}=8-\operatorname{deg} \Delta$ since $F_{1}$ is a conic bundle with deg $\Delta$ singular fibres over a line in $\mathbb{P}^{2}$. On the other hand, $\left(-K_{F_{1}}\right)^{2}=$ $\left(-K_{Y^{\prime}}-F_{1}\right)^{2} F_{1}=\left(B-F_{1}\right)^{2} F_{1}=B^{2} F_{1}-2 B\left(F_{1}\right)^{2}$. Since $\left(F_{1}\right)^{2}$ is numerically equivalent to a fibre $l$ of $Y^{\prime} \rightarrow \mathbb{P}^{2}$, we have $B\left(F_{1}\right)^{2}=-K_{Y^{\prime}} l=2$. Thus the degree of the discriminant is equal to $\operatorname{deg} \Delta=12-B^{2} F_{1}$ when computed on $Y^{\prime}$; this is standard, seen already in [MM82], for example.

We can equally well compute on $Y$, since the map $Y \rightarrow Y^{\prime}$ is a $B$-flop. Computing on $Y$, we see that $A E^{2}=A^{2} E=0$, so

$$
\begin{aligned}
B^{2} F_{1} & =\left(A-\frac{1}{r} E\right)^{2}\left(a_{1} A-\left(\frac{a_{1}+r}{r}\right) E\right) \\
& =a_{1} A^{3}-\left(\frac{a_{1}+r}{r^{3}}\right) E^{3} \\
& =a_{1} A^{3}-\left(\frac{a_{1}+r}{r}\right)\left(\frac{1}{a(r-a)}\right)
\end{aligned}
$$

as required.

In fact, only the following three links from the 69 families result in a conic bundle:

Family No. 2. Here $X \subset \mathbb{P}^{6}(1,1,1,1,1,1,2)$, which has degree $13 / 2$ and basket $\{1 / 2(1,1,1)\}$. (The equations of $X$ have degrees $2,3,3,3,3$ and $Y \rightarrow$ $Y^{\prime}$ flops seven irreducible rational curves.)

Thus $a_{1}=a_{2}=a_{3}=1$ and the discriminant has degree

$$
\operatorname{deg} \Delta=12-13 / 2+3 / 2=7 \text {. }
$$


FAmily No. 3. Here $X \subset \mathbb{P}^{6}(1,1,1,1,1,2,3)$, which has degree $14 / 3$ and basket $\{1 / 3 \times(1,1,2)\}$. (The equations of $X$ have degrees $3,3,4,4,4$ and $Y \rightarrow Y^{\prime}$ flops six irreducible rational curves.)

Thus $a_{1}=a_{2}=a_{3}=1$ and the discriminant has degree

$$
\operatorname{deg} \Delta=12-14 / 3+4 / 3 \times 1 / 2=8 \text {. }
$$

FAmily No. 8. Here $X \subset \mathbb{P}^{6}(1,1,1,2,2,2,2)$, which has degree $5 / 2$ and basket $\{5 \times 1 / 2(1,1,1)\}$. (The equations of $X$ have degrees $4,4,4,4,4$ and $Y \rightarrow Y^{\prime}$ flops 12 irreducible rational curves.)

So $a_{1}=a_{2}=a_{3}=2$ and the discriminant has degree

$$
\operatorname{deg} \Delta=12-2 \times 5 / 2+4 / 2=9 .
$$

Proposition 3.13 (Del Pezzo fibration). If $a_{1}=a_{2}<a_{3}$, then the degree of fibres of the del Pezzo fibration $Y^{\prime} \rightarrow \mathbb{P}^{1}$ is

$$
\left(-K_{F_{1}}\right)^{2}=\frac{1}{\left(a_{3}-a_{1}\right)^{2}}\left(a_{1} a_{3}^{2} \operatorname{deg}(X)-\frac{\left(a_{1}+r\right)\left(a_{3}+r\right)^{2}}{r a(r-a)}+N\right),
$$

where $N$ is the number of curves flopped by $Y_{1} \rightarrow Y^{\prime}$, which is the same number $N$ computed in Corollary 3.4.

Proof. Computing on $Y_{2}$, we choose $F_{1}$ as a fibre to work on and find its degree as

$$
\begin{aligned}
\left(-K_{F_{1}}\right)^{2}=\left(B-F_{1}\right)_{\mid F_{1}}^{2}=B^{2} F_{1} & =\frac{1}{\left(a_{3}-a_{1}\right)^{2}}\left(F_{3}-F_{1}\right)^{2} F_{1} \\
& =\frac{1}{\left(a_{1}-a_{3}\right)^{2}} F_{1} F_{3}^{2},
\end{aligned}
$$

where equalities come from $F_{1}^{2}=0$ on $Y_{2}$. We compute this number on $X$.

The flip $Y_{1} \rightarrow Y_{2}$ is an $F_{3}$-flop, so the expression $F_{1} F_{3}^{2}$ is the same whether computed on $Y_{1}$ or $Y_{2}$. The flop $Y \rightarrow Y_{1}$ is a true $B$-flop, so the expression $F_{1} F_{3}^{2}=F_{1}\left(F_{2}+\left(a_{3}-a_{2}\right) B\right) F_{3}$ differs only in the term $F_{1} F_{2} F_{3}$ when computed on $Y$ rather than $Y_{1}$. This intersection defines exactly the reduced flopping curves, which are contracted to nonsingular points on $F_{1}$ considered in $Y_{1}$. So $F_{1} F_{3}^{2}$ computed on $Y_{1}$ equals $F_{1} F_{3}^{2}+N$ computed on $Y$.

Now $F_{i}=a_{i} B-E=a_{i} A-\left(\left(a_{i}+r\right) / r\right) E$, so $A E^{2}=A^{2} E=0$ and $E^{3}=$ $r^{2} /(a(r-a))$ completes the calculation. 
Table 1: Sarkisov links that result in a del Pezzo fibration. The link comprises the Kawamata blowup of the Type I centre $p \in X$ followed by the standard flop of the indicated number of curves, completed by a flip to the total space of the fibration.

\begin{tabular}{cccccrrr}
\hline No. & Fano 3-fold $X$ & $p \in X$ & $A^{3}$ & $a_{3}, a_{2}, a_{1}$ & $\#$ & {$[n]$} & $d$ \\
\hline 4 & $X_{3^{4}, 4} \subset \mathbb{P}\left(1^{5}, 2^{2}\right)$ & $\frac{1}{2}(1,1,1)$ & 5 & $2,1,1$ & 8 & {$[2]$} & 4 \\
6 & $X_{4^{2}, 5^{2}, 6} \subset \mathbb{P}\left(1^{4}, 2,3,4\right)$ & $\frac{1}{4}(1,1,3)$ & $11 / 4$ & $2,1,1$ & 7 & {$[3]$} & 3 \\
7 & $X_{3,4^{3}, 5} \subset \mathbb{P}\left(1^{4}, 2,2,3\right)$ & $\frac{1}{3}(1,1,2)$ & $19 / 6$ & $2,1,1$ & 7 & {$[2]$} & 3 \\
10 & $X_{4^{3}, 5^{2}} \subset \mathbb{P}\left(1^{3}, 2^{3}, 3\right)$ & $\frac{1}{2}(1,1,1)$ & $13 / 6$ & $3,2,2$ & 14 & {$[3]$} & 3 \\
12 & $X_{4,5,6^{2}, 7} \subset \mathbb{P}\left(1^{3}, 2^{2}, 3,5\right)$ & $\frac{1}{5}(1,2,3)$ & $17 / 10$ & $2,1,1$ & 5 & {$[2]$} & 2 \\
16 & $X_{4,5^{2}, 6^{2}} \subset \mathbb{P}\left(1^{2}, 2^{3}, 3^{2}\right)$ & $\frac{1}{3}(1,1,2)$ & $7 / 6$ & $3,2,2$ & 11 & {$[2]$} & 2 \\
17 & $X_{6^{2}, 7^{2}, 8} \subset \mathbb{P}\left(1^{2}, 2^{2}, 3,4,5\right)$ & $\frac{1}{5}(1,1,4)$ & $4 / 5$ & $3,2,2$ & 10 & {$[4]$} & 2 \\
21 & $X_{6^{3}, 7^{2}} \subset \mathbb{P}\left(1^{2}, 2,3^{3}, 4\right)$ & $\frac{1}{3}(1,1,2)$ & $3 / 4$ & $4,3,3$ & 15 & {$[4]$} & 2 \\
43 & $X_{5,6^{3}, 7} \subset \mathbb{P}\left(1^{2}, 2^{2}, 3^{2}, 4\right)$ & $\frac{1}{4}(1,1,3)$ & $11 / 12$ & $3,2,2$ & 10 & {$[3]$} & 2 \\
55 & $X_{8,9,10^{2}, 11} \subset \mathbb{P}\left(1,2,3^{2}, 4,5,7\right)$ & $\frac{1}{7}(1,2,5)$ & $3 / 14$ & $4,3,3$ & 8 & {$[2]$} & 1 \\
56 & $X_{8,9^{2}, 10^{2}} \subset \mathbb{P}\left(1,2,3,4^{2}, 5^{2}\right)$ & $\frac{1}{5}(1,2,3)$ & $1 / 5$ & $5,4,4$ & 11 & {$[2]$} & 1 \\
\hline
\end{tabular}

Notation: \# denotes the number of flopped curves; $A^{3}$ is the degree of $X ; d$ is the degree of the del Pezzo fibre. The column $[n]$ describes the flip: $[n]$ indicates the toric hypersurface flip of $n-1$ irreducible $\mathbb{P}^{1} \mathrm{~s}$ meeting in the quotient singularity $(1 / n)(1,1, n-1)$ flipped to a single curve passing through no singularities (see the classification in [Bro99, Theorem 8.1(I)(1)] with $a_{1}=n$ and $\left.a_{2}=b_{1}=1\right)$.

In fact, 11 of the links from the 69 families result in del Pezzo fibrations. We list them in Table 1. The description of the del Pezzo fibration is rather coarse, giving only the degree of the general fibre according to Proposition 3.13. Computing the link in detail, using the methods of Section 5.2.1, for instance, one sees that some of these fibrations are standard in a neighbourhood of each fibre in the sense of Corti [Cor96, Definitions 1.8 and 1.13]. For instance, the cubic fibration resulting from Fano family no. 6 is embedded as a hypersurface in a $\mathbb{P}^{3}$ scroll over $\mathbb{P}^{1}$ : the transforms of the variables of weights 3 and 4 can be eliminated in every fibre if the equations of $X$ are sufficiently general. In the notation of [BCZ04, Table 2 and Section 4.4.3], this link is the reverse of the link $8 \mathrm{a}-\mathrm{a}$ link arising as a special member of the linear system $|3 M-L|$ on $\mathbb{F}(1,1,2)$. Family no. 17 gives another example: the end of the link is a relative degree 4 hypersurface in a $\mathbb{P}(1,1,1,2)$ scroll over $\mathbb{P}^{1}$. These are the only two such straightforward cases whose resulting fibration is a hypersurface. 
In contrast, the link from Fano family no. 7 (and equally no. 10) results in a cubic fibration described as a relative $(2,3)$ complete intersection $Y_{2,3}^{\prime}$ in a $\mathbb{P}\left(1^{4}, 2\right)$ scroll over $\mathbb{P}^{1}$ : the equation of degree 2 eliminates the weight 2 fibre variable in most fibres, leaving only the cubic, but in one of the fibres (or two in the case of no. 10) it does not. This is to be expected from a count of the singularities as follows. The general element $X_{3,4^{3}, 5}$ of family no. 7 has singularities $1 / 3(1,1,2)$ and $1 / 2(1,1,1)$. The initial Kawamata blowup of the index 3 point leaves $2 \times 1 / 2(1,1,1)$, the flop leaves those unchanged, and the final Francia flip removes one of these quotient singularities. The resulting cubic fibration is not Gorenstein and so does not appear in [BCZ04, Table 2] — the analysis there starts with a Gorenstein model. From the point of view of rigidity and Corollary 1.2, this is enough, since the end of the link is certainly a different Mori fibre space. However, one can simplify the model of the cubic fibration in a neighbourhood of the bad fibre following Corti [Cor96] and Kollár [Kol97]: after blowing up the remaining 1/2(1,1,1) singularity and contracting a divisor, the result is an element of the linear system $|3 M-2 L|$ on $\mathbb{F}(1,1,2)$. This identifies the square birational type of the cubic fibration in the geography of [BCZ04], but nothing more is gained, for all elements of that system are already known to be nonrigid following the flop of the negative section into a Type III link.

\section{$\S 4$. The 2-ray game for toric varieties}

The following result is well known: it follows from [HK00, Corollary 2.10], for instance, or equally from both [Rei83] and [FS04]. To get detailed information about the geometry of each step, which we apply in Section 5, we trace a proof with an explicit commentary. Note that there are no conditions on the singularities of $\mathcal{X}$ or $\mathcal{Y}$ in this theorem.

Theorem 4.1. Let $\mathcal{X}$ be a $\mathbb{Q}$-factorial toric variety with $\rho \mathcal{X}=1$, and let $\mathcal{Y}_{0} \rightarrow \mathcal{X}$ be the contraction of a divisor corresponding to the inclusion of a new element in the 1-skeleton of the fan $\Delta_{\mathcal{X}}$. Then there is a unique sequence of projective toric maps:

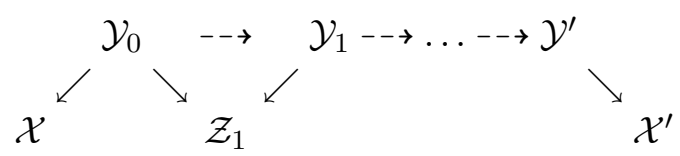

At each step, the map $\mathcal{Y}_{i \rightarrow-} \rightarrow \mathcal{Y}_{i+1}$ is an isomorphism in codimension 1. The morphism $\mathcal{Y}^{\prime} \rightarrow \mathcal{X}^{\prime}$ is either a divisorial contraction or a fibration to a toric variety of lower dimension. 


\subsection{The Cox ring and the proof of Theorem 4.1}

We describe the 2-ray game for a rank 2 toric variety in terms of its homogeneous coordinate ring. Compare the explicit calculations with Cox's description of the homogeneous coordinate ring in [Cox95] and with [Rei97, Lecture 2].

4.1.1. A rank 2 torus action. Let $R_{0}=\mathbb{C}\left[x_{1}, \ldots, x_{N}\right]$, and let $\mathcal{A}=\operatorname{Spec} R_{0}$. Let $G=\mathbb{C}^{*} \times \mathbb{C}^{*}$ with coordinates $\lambda, \mu$. An action of $G$ on $\mathcal{A}$ is determined by $N$ characters,

$$
\chi_{i} \in C_{G}=\operatorname{Hom}_{\mathbb{Z}}\left(G, \mathbb{C}^{*}\right), \quad \text { for } i=1, \ldots, N,
$$

by defining $g^{*}\left(x_{i}\right)=\chi_{i}(g) \cdot x_{i}$ for $i=1, \ldots, N$. Of course, the isomorphism $\mathbb{Z} \oplus \mathbb{Z} \rightarrow C_{G}$ taking $(a, b)$ to the character $(\lambda, \mu) \mapsto \lambda^{a} \mu^{b}$ makes this explicit: if $\chi_{i}$ corresponds to $\left(a_{i}, b_{i}\right)$, then $(\lambda, \mu) \in G$ acts on $\mathcal{A}$ by

$$
\left(x_{1}, \ldots, x_{n}\right) \mapsto\left(\lambda^{a_{1}} \mu^{b_{1}} x_{1}, \ldots, \lambda^{a_{N}} \mu^{b_{N}} x_{N}\right) .
$$

We leave this isomorphism implicit for the remainder of this article, and we write $\chi_{i}=\left(a_{i}, b_{i}\right)$. Once and for all, we fix such an action: we fix a choice of $N$ characters $\chi_{i}=\left(a_{i}, b_{i}\right) \in \mathbb{Z} \oplus \mathbb{Z}$, and we insist that these characters rationally span $\mathbb{Z} \oplus \mathbb{Z}$.

In order to apply GIT, we consider the trivial line bundle $\mathcal{L} \rightarrow \mathcal{A}$, with fibre coordinate $t$, by the embedding $R_{0} \subset R=\mathbb{C}\left[x_{1}, \ldots, x_{N}, t\right]$. To extend the action compatibly from $\mathcal{A}$ to $\mathcal{L}$ is just a matter of choosing another character $\chi \in C_{G}$ and defining

$$
g^{*}(t)=\chi(g)^{-1} \cdot t
$$

As usual, we regard $\chi$ as a parameter, and we emphasise this choice in the notation by writing $g^{*, \chi}$ for the action of $g \in G$ on $\mathcal{L}$. Again, we can be more explicit: if $\chi$ corresponds to $(a, b) \in \mathbb{Z} \oplus \mathbb{Z}$, then $(\lambda, \mu) \in G$ acts on $\mathcal{L}$ by

$$
\left(x_{1}, \ldots, x_{n}\right) \mapsto\left(\lambda^{a_{1}} \mu^{b_{1}} x_{1}, \ldots, \lambda^{a_{N}} \mu^{b_{N}} x_{N}, \lambda^{-a} \mu^{-b} t\right) .
$$

We denote the invariant ring under this action on $\mathcal{L}$ by

$$
R^{G, \chi}=\left\{f \in R: g^{*, \chi}(f)=f\right\} .
$$

If $f \in R^{G, \chi}$, then we define $D_{f}=\{P \in \mathcal{L}: f(P) \neq 0\}$. A point $\left(p_{1}, \ldots, p_{N}\right) \in$ $\mathcal{A}$ is $\chi$-semistable if and only if there exists $f \in R^{G, \chi}$, with $f \notin R_{0}$, and $s \in \mathbb{C}^{*}$ such that $\left(p_{1}, \ldots, p_{N}, s\right) \in D_{f}$. The set of $\chi$-semistable points is denoted $\mathcal{A}_{\chi}^{\text {ss }}$. 
Finally, the GIT quotient of $\mathcal{A}$ by $G$ (associated to the choice of $\chi$ ) is denoted $\mathcal{A} / /{ }_{\chi} G$ and is defined to be the categorical quotient $\mathcal{A}_{\chi}^{\text {ss }} / / G$. As explained in [Dol03, Theorem 8.1], this quotient is locally of the form $D_{f} / / G=\operatorname{Spec} \mathcal{O}\left(D_{f}\right)^{G}$, where $f$ is a $G$-equivariant function and $D_{f}$ is the corresponding principal open affine set.

4.1.2. Projective description of the quotient. Each choice of $\chi$ determines a $\mathbb{Z} \oplus \mathbb{Z}$ grading on $R$ as described above. It is convenient to introduce another grading on $R$ (and its subrings) to carry line bundles on the various quotients. The new $\mathbb{N}$ grading is by powers of $t$ or, equivalently, by setting $\operatorname{deg}\left(x_{i}\right)=0$ for each $i$ and $\operatorname{deg}(t)=1$. We denote the piece of $R$ in degree $m \geq 0$ by $R_{m}$ (notation consistent with the earlier use of $R_{0} \subset R$ ). Clearly, this grading survives taking invariants: $\left(R^{G, \chi}\right)_{m}=\left(R_{m}\right)^{G, \chi}$ for each degree $m \geq 0$.

We can form Proj $R$ with respect to this grading, and it can be regarded as the $\mathbb{C}^{*}$ quotient

$$
\pi: \operatorname{Spec} R \backslash \operatorname{Spec} R_{0} \rightarrow \operatorname{Proj} R \text {. }
$$

Of course, $\operatorname{Proj} R=\mathcal{A}=\operatorname{Spec} R_{0}$ is affine in this case, and the map $\pi$ above is just the restriction away from the zero section of the projection $\mathcal{L} \rightarrow \mathcal{A}$, which is a geometric quotient. We can do the same for subrings of $R$, and we denote the quotient maps corresponding to any $\chi$ by

$$
\pi^{G, \chi}: \operatorname{Spec} R^{G, \chi} \backslash \operatorname{Spec} R_{0}^{G, \chi} \rightarrow \operatorname{Proj} R^{G, \chi} .
$$

The degree 0 inclusion $R^{G, \chi} \rightarrow R$ induces maps $\rho$ below:

$$
\begin{aligned}
& \operatorname{Spec} R \backslash \operatorname{Spec} R_{0} & \stackrel{\rho}{\longrightarrow} & \operatorname{Spec} R^{G, \chi} \backslash \operatorname{Spec} R_{0}^{G, \chi} \\
& \downarrow \pi & & \downarrow \pi^{G, \chi} \\
\mathcal{A}_{\chi}^{\mathrm{ss}} \stackrel{j}{\hookrightarrow} & \operatorname{Proj} R & -\rho & \operatorname{Proj} R^{G, \chi}
\end{aligned}
$$

Proposition 4.2. The map $\rho \circ j=\pi_{\chi}: \mathcal{A}_{\chi}^{\mathrm{ss}} \rightarrow \operatorname{Proj} R^{G, \chi}$ is a surjective morphism and it induces an isomorphism $\mathcal{A} / / \chi_{\chi} G \cong \operatorname{Proj} R^{G, \chi}$.

Proof. Both the surjectivity of $\pi_{\chi}$ and the fact that it is defined at geometric points are straightforward: at a point in $\operatorname{Proj} R^{G, \chi}$ there is a (nontrivial, invariant) function that does not vanish there, which is the same as the condition for semistability. Indeed, $\pi_{\chi}$ is expressed in coordinates as a choice of generators for $R^{G, \chi}$ as an $R_{0}^{G, \chi}$-algebra, and a point lies in $\mathcal{A}_{\chi}^{\text {ss }}$ if and only if some polynomial in these generators does not vanish there. 
To check that $\pi_{\chi}$ is set-theoretically injective, consider $p_{1}, p_{2} \in \mathcal{A}$ lying in $G$-orbits whose closures are disjoint. There is an invariant function $f$ which vanishes on the orbit $G \cdot p_{1}$ and is not identically zero on $G \cdot p_{2}$. This function then separates the image of the points on $\operatorname{Proj} R^{G, \chi}$.

Finally, we match scheme structures by considering local patches. An affine patch on Proj $R^{G, \chi}$ is Spec of the ring of homogeneous rational functions of total degree 0 in $t$ which have expressions only in $f$ in the denominator. But this ring is exactly $\mathcal{O}_{D_{f}}^{G}$, and its spectrum is an affine patch on $\mathcal{A} / /{ }_{\chi} G$ by construction of the quotient.

4.1.3. Notation: The effective cone of characters. The characters $\chi_{i}: G \rightarrow$ $\mathbb{C}^{\star},(\lambda, \mu) \mapsto \lambda^{a_{i}} \mu^{b_{i}}, i=1, \ldots, N$ span a convex cone denoted $\Sigma_{\text {eff }} \subset C_{G} \otimes \mathbb{R} \cong$ $\mathbb{R}^{2}$. Let $r_{0}, \ldots, r_{M} \subset \Sigma_{\text {eff }}$ be the distinct rays generated by the characters $\chi_{i} \in \mathbb{R}^{2}$. Reordering if necessary, we may assume that the rays $r_{\alpha}$ are ordered clockwise from one boundary of $\Sigma_{\text {eff }}$ to the other. Two characters can generate the same ray, and so we may assume, again after reordering, for each $r_{\alpha}$ there are integers $l_{\alpha} \leq m_{\alpha}$ such that

$$
\chi_{i} \in r_{\alpha} \text { if and only if } l_{\alpha} \leq i \leq m_{\alpha}
$$

The collection of rays $\left\{r_{0}, \ldots, r_{M}\right\}$ divides $\Sigma_{\text {eff }}$ into $M$ chambers and we denote by $\mathcal{C}_{\alpha}$ these open chambers; in particular, $\partial \overline{\mathcal{C}_{\alpha}}=r_{\alpha} \cup r_{\alpha+1}, \alpha=$ $0, \ldots, M-1$ :

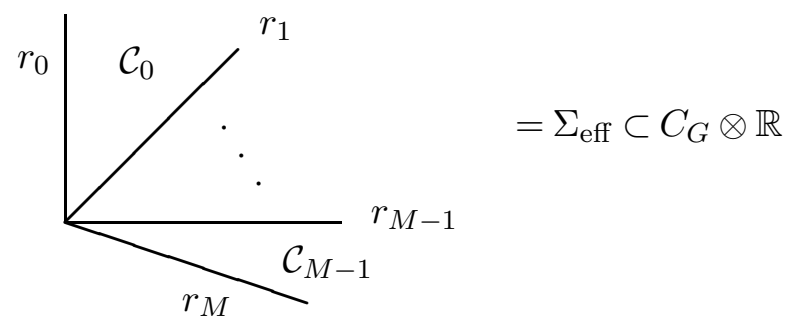

For the rest of this article, a character $\chi$ is identified with the corresponding $(a, b) \in \mathbb{R}^{2}$ by the isomorphism $C_{G} \otimes \mathbb{R} \cong \mathbb{R}^{2}$ which sends $\chi \mapsto(a, b)$. For any two rays $r, r^{\prime} \subset \Sigma_{\text {eff }}$ (not necessarily among the $r_{i}$ ) we say that $r \leq r^{\prime}$ if and only if $r$ is contained in the convex span of $r_{0}$ and $r^{\prime}$. We extend this partial order to elements of $C_{G}$ by saying $(a, b) \leq\left(a^{\prime}, b^{\prime}\right)$ when $(a, b) \mathbb{R}_{\geq 0} \leq\left(a^{\prime}, b^{\prime}\right) \mathbb{R}_{\geq 0}$ (i.e., we simply put elements of $\Sigma_{\text {eff }}$ in clockwise partial order). 
4.1.4. Stability and geometric quotients. Let $(a, b) \in \Sigma_{\text {eff }}$. We define a $G$ invariant open affine subset $\mathcal{B}_{(a, b)}=\mathcal{A} \backslash V\left(J_{(a, b)}\right) \subset \mathcal{A}$, where $J_{(a, b)} \subset R_{0}$ is the homogeneous ideal

$$
J_{(a, b)}=\left(\left\{x_{i}:\left(a_{i}, b_{i}\right) \leq(a, b)\right\}\right) \cap\left(\left\{x_{i}:\left(a_{i}, b_{i}\right) \geq(a, b)\right\}\right)
$$

and where $x_{1}, \ldots, x_{N}$ are the coordinates on $\mathcal{A}$. (Note that $\left(\left\{x_{i}:\left(a_{i}, b_{i}\right) \leq\right.\right.$ $(a, b)\}) \subset R_{0}$ is the ideal generated by those $x_{i}$ on the anticlockwise side of $(a, b)$ in $\left.\Sigma_{\text {eff }}\right)$

Recall, from [Dol03, (8.1)] for instance, that a point $p \in \mathcal{A}$ is $\chi$-stable if and only if the following three conditions hold: it is $\chi$-semistable, its stabiliser $G_{p} \subset G$ is finite, and, if $f \in R^{G, \chi}$ is as in the definition of $\chi_{\text {- }}$ semistable, then for every $q \in D_{f}$ the orbit $G \cdot q$ is closed in $D_{f}$.

Proposition 4.3. Fix a character $\chi$ corresponding to a primitive integral vector $(a, b) \in \Sigma_{\text {eff }} \cap C_{G}$. Then $\mathcal{A}_{\chi}^{\text {ss }}=\mathcal{B}_{(a, b)}$. If, moreover, $(a, b)$ is in the interior of some chamber $\mathcal{C}_{j}$, then $\mathcal{A}_{\chi}^{\mathrm{s}}=\mathcal{A}_{\chi}^{\mathrm{ss}}$, so the quotient $\mathcal{A} / /{ }_{\chi} G$ is geometric.

Proof. A point $p \in \mathcal{A}$ lies in $\mathcal{B}_{(a, b)}$ if there exist both a variable $x_{i}$ with $\left(a_{i}, b_{i}\right) \leq(a, b)$ for which $x_{i}(p) \neq 0$ and another $x_{j}$ (possibly equal to $x_{i}$ ) with $\left(a_{j}, b_{j}\right) \geq(a, b)$ for which $x_{j}(p) \neq 0$. Let $m, m_{i}, m_{j} \in \mathbb{N}$ such that $m(a, b)=$ $m_{i}\left(a_{i}, b_{i}\right)+m_{j}\left(a_{j}, b_{j}\right)$. If $F=x_{i}^{m_{i}} x_{j}^{m_{j}} t^{m}$, then $F \in R_{m}^{G_{\chi}}$ and $F(p, 1) \neq 0$. In particular, $p \in \mathcal{A}_{\chi}^{\mathrm{ss}}$ and $\mathcal{B}_{(a, b)} \subset \mathcal{A}_{\chi}^{\mathrm{ss}}$.

Conversely, if $p \in \mathcal{A}_{\chi}^{\mathrm{ss}}$, then for some $m>0$ there exist $F \in R_{m}^{G_{\chi}}$ and $\tau \in \mathbb{C}^{\star}$ such that $F(p, \tau) \neq 0$. Any monomial appearing in $F$ is of the form $x_{1}^{i_{1}} \cdots x_{N}^{i_{N}} t^{m}$. After evaluating $t$ at $\tau$, such a monomial is in $J_{(a, b)}$, so $F_{\mid t=\tau}$ is also in $J_{(a, b)}$. Since $F_{\mid t=\tau}(p) \neq 0, \mathcal{A}_{\chi}^{\text {ss }} \subset \mathcal{B}_{(a, b)}$ and the two sets are equal.

Suppose now that $\chi=(a, b)$ is the interior of some chamber. We show that $\mathcal{A}_{\chi}^{\mathrm{ss}} \subset \mathcal{A}_{\chi}^{\mathrm{s}}$. Let $p \in \mathcal{A}_{\chi}^{\mathrm{ss}}=\mathcal{B}_{(a, b)}$. Fix $i, j$ such that the two components $x_{i}(p), x_{j}(p)$ are nonzero and

$$
\left(a_{i}, b_{i}\right)<(a, b)<\left(a_{j}, b_{j}\right)
$$

In particular, $D_{f} \subset \mathcal{A}_{\chi}^{\text {ss }}$ for $f=x_{i} x_{j}$. If $(\lambda, \mu) \in G_{p}$, then $\lambda^{a_{i}} \mu^{b_{i}}=1$ and $\lambda^{a_{j}} \mu^{b_{j}}=1$; these equations have only finitely many solutions, so $G_{p}$ is finite. By the same token, $G_{q}$ is finite for any $q \in D_{f}$. Thus all orbits in this set are closed, and $p \in \mathcal{A}_{\chi}^{\mathrm{s}}$. 
4.1.5. Affine patches on the quotients. It is convenient to record here the natural affine patches on these geometric quotients, although we do not need them yet. Suppose that $\chi$ corresponds to $(a, b)$ and does not lie on any ray $r_{\alpha}, \alpha=1, \ldots, M$. Then, by Proposition 4.3,

$$
\mathcal{A}_{i, j}=\mathcal{A} \backslash\left(x_{i} x_{j}=0\right),
$$

for any $i<j$ with $\left(a_{i}, b_{i}\right)<(a, b)<\left(a_{j}, b_{j}\right)$, is an open affine $G$-invariant set contained in $\mathcal{A}_{\chi}^{\text {ss }}$, and moreover, $\mathcal{A}_{\chi}^{\text {ss }}$ is covered by such sets $\mathcal{A}_{i, j}$.

If the two characters $\left(a_{i}, b_{i}\right)$ and $\left(a_{j}, b_{j}\right)$ are a $\mathbb{Z}$-basis for $\mathbb{Z} \oplus \mathbb{Z}$, then the usual identification of affine patches on $\mathbb{P}^{n}$ with $\mathbb{C}^{n}$ shows that $\mathcal{A}_{i, j} / /{ }_{\chi} G \cong$ $\mathbb{C}^{N-2}$ and that the variables $x_{1}, \ldots, \widehat{x}_{i}, \ldots, \widehat{x}_{j}, \ldots, x_{N}$ (omitting $x_{i}$ and $x_{j}$ ) serve as coordinates on this patch: the two degrees of freedom in the group action can be used to fix values for the $i$ th and $j$ th coordinates of a point, leaving the other coordinates free. If instead $\left(a_{i}, b_{i}\right)$ and $\left(a_{j}, b_{j}\right)$ span a sublattice of $C_{G}$ of index $r$, then the patch is a $\mathbb{Z} / r \mathbb{Z}$ quotient of $\mathbb{C}^{N-2}$ in the same way as for patches on WPS.

4.1.6. Extremal morphisms between the quotients. Fix a chamber $\mathcal{C}_{\alpha}$ with its boundary rays $r_{\alpha}$ and $r_{\alpha+1}$. By Proposition 4.3, the polarisations $\chi \in \mathcal{C}_{\alpha}$ give isomorphic geometric quotients (by well-defined isomorphisms), so set

$$
\mathcal{Y}_{\alpha}=\operatorname{Proj} R^{G, \chi} \quad \text { for some } \chi \in \mathcal{C}_{\alpha} .
$$

Equally, the strictly semistable polarisations $(a, b) \in r_{\alpha}$ give isomorphic categorical quotients, so set

$$
\mathcal{Z}_{\alpha}=\operatorname{Proj} R^{G, \chi} \text { for some } \chi \in r_{\alpha} .
$$

Lemma 4.4. Let $\alpha \in\{0, \ldots, M-1\}$.

(a) Here $\mathcal{Y}_{\alpha}$ is $\mathbb{Q}$-factorial of dimension $N-2$ and Picard rank $\rho \leq 2$.

(b) Here $\mathcal{Y}_{\alpha}$ admits two morphisms

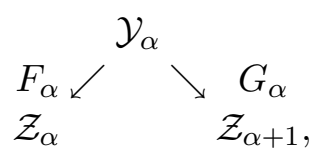

either of which may be an isomorphism.

The task in (b) is to describe the degree 0 homomorphism of graded rings that is induced by the inclusion map of subsets of $\mathcal{A}$. We take advantage of the freedom in which graded ring we actually use as the homogeneous coordinate ring of $\mathcal{Y}_{\alpha}$. 
Proof. (a) The dimension count $\operatorname{dim} \mathcal{Y}_{\alpha}=\operatorname{dim} \mathcal{A}_{\chi}^{\mathrm{ss}}-\operatorname{dim} G$ holds because the quotient is geometric. The $\mathbb{Q}$-factoriality follows from [HK00, Lemma 2.1]; to confirm the hypotheses of that Lemma, invariant Cartier divisors on $\mathcal{A}_{\chi}^{\mathrm{s}}$ are supported on the hyperplanes $x_{i}=0$ and so can be linearised as required. Again following [HK00, Lemma 2.1], the map

$$
\operatorname{Pic}\left(\mathcal{Y}_{\alpha}\right)_{\mathbb{Q}} \longrightarrow \operatorname{Pic}^{G}\left(\mathcal{A}_{\chi}^{\mathrm{s}}\right)_{\mathbb{Q}}
$$

is an isomorphism. If there is exactly one character lying strictly on one side of $r_{\alpha} \subset \Sigma_{\text {eff }}$-that character would have to be either $\chi_{0}$ or $\chi_{N}$-then the codomain of this map is $\mathbb{Q}$. Otherwise, there are at least two characters on each side of $r_{\alpha}$, and the codomain is $\mathbb{Q}^{2}$.

(b) We describe $G_{0}: \mathcal{Y}_{0} \rightarrow \mathcal{Z}_{1}$; other cases are similar. Choose characters $\chi_{0}, \chi_{1}$ lying in the rays $r_{0}, r_{1}$, respectively. Pick a set of generators $f_{1}, \ldots, f_{v}$ of $R_{1}=R^{G, \chi_{1}}$, the homogeneous coordinate ring of $\mathcal{Z}_{1}$. Let $g \in R_{m}^{G, \chi_{0}}$ be any nonzero polynomial of positive degree $m>0$ (in $t$ ) in the homogeneous coordinate ring of $\mathcal{Z}_{0}$.

The elements $F_{i}=f_{i} g^{\operatorname{deg}_{t}\left(f_{i}\right)}$ all lie in $R=R^{G, \chi}$, where $\chi=\chi_{0}^{m} \chi_{1}$ lies in the interior of the chamber $\mathcal{C}_{0}$. So we use (some truncation of) $R$ as the homogeneous coordinate ring of $\mathcal{Y}_{0}$, and the map $R_{1} \rightarrow R$ induced by $f_{i} \mapsto F_{i}$ is the required degree 0 graded $k$-algebra homomorphism.

4.1.7. Divisors on the quotients and exceptional loci. For a homogeneous element $x \in R_{0}$ define the basic divisor $\mathcal{D}_{x}$ to be the divisor $x=0$ in each quotient. (The notation is birational, and the variety on which $\mathcal{D}_{x}$ is currently being considered will be mentioned explicitly if it matters. Of course, $\mathcal{D}_{x}$ only makes sense on quotients $\mathcal{A}_{\chi}^{\text {ss }} / / G$ for which $x$ does not generate a component of the irrelevant ideal.)

Intersections of basic divisors describe the base locus of linear systems. Define

$$
\mathcal{F}_{\alpha}=\bigcap_{\chi_{i}<r_{\alpha-1}} \mathcal{D}_{x_{i}} \subset \mathcal{Y}_{\alpha} \quad \text { and } \quad \mathcal{G}_{\alpha}=\bigcap_{\chi_{i}>r_{\alpha+1}} \mathcal{D}_{x_{i}} \subset \mathcal{Y}_{\alpha} .
$$

(As usual, $\chi_{i}$ is the character associated to variable $x_{i}$.) Also, let

$$
\mathcal{B}_{\alpha}=\bigcap_{\chi_{i} \in r_{\alpha}} \mathcal{D}_{x_{i}} \subset \mathcal{Z}_{\alpha}
$$

The maps $F_{\alpha}$ and $G_{\alpha}$ from $\mathcal{Y}_{\alpha}$ are defined in Lemma 4.4. 
Lemma 4.5. Let $\alpha \in\{0, \ldots, M-1\}$.

(a) The exceptional locus of the morphism $F_{\alpha}$ is $\mathcal{F}_{\alpha}$, and the restriction of $F_{\alpha}$ to the exceptional locus $F_{\alpha}: \mathcal{F}_{\alpha} \rightarrow \mathcal{B}_{\alpha}$ exhibits $\mathcal{F}_{\alpha}$ as a weighted projective bundle over $\mathcal{B}_{\alpha}$. Similarly, $G_{\alpha}: \mathcal{G}_{\alpha} \rightarrow \mathcal{B}_{\alpha+1}$ is a weighted projective bundle over $\mathcal{B}_{\alpha+1}$.

(b) The map $\mathcal{Y}_{\alpha} \rightarrow-\rightarrow \mathcal{Y}_{\alpha+1}$ in the diagram

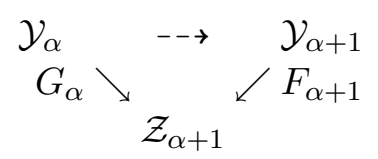

is a $\mathcal{D}_{x}$-flop for any $x$ whose character lies in the ray $r_{\alpha}$.

Proof. We prove the requirements for $F_{\alpha}$. To prove (a), consider first the case $F_{0}: \mathcal{Y}_{0} \rightarrow \mathcal{Z}_{0}$. In this case, $\mathcal{Z}_{0}$ coincides with $\mathcal{B}_{0}$ and is a WPS (possibly just a point), and the map $F_{0}$ has effective $\mathbb{C}^{*}$ quotients of affine space punctured at the origin as its fibres. The general case follows, since $F_{\alpha}: \mathcal{F}_{\alpha} \rightarrow \mathcal{B}_{\alpha}$ is of the same form as the $F_{0}$ just considered.

To prove (b), note that any such $x$ has as its zero locus a divisor in $\mathcal{B}_{\alpha}$ and so is certainly trivial on the fibres of $F_{\alpha}$. The exceptional loci are irreducible, so the relative Picard rank is one.

4.1.8. Localisation and flips. To understand the birational map $\mathcal{Y}_{\alpha}-\rightarrow \mathcal{Y}_{\alpha+1}$ better, we realise it as a variation of $\mathbb{C}^{*}$ action in a neighbourhood of a point in the base $\mathcal{Z}_{\alpha}$. If $x_{l}, x_{l+1}, \ldots, x_{l+k}$ are the variables whose characters lie in $r_{\alpha}$, we consider the case of the point $x_{l+1}=\cdots=x_{l+k}=0$ in $\mathcal{B}_{\alpha} \subset \mathcal{Z}_{\alpha}$ and that $\chi_{l}$ is a primitive vector in $C_{G}$. (This is not quite the general case, but in our applications it is enough.) The quotient map $C_{G} \rightarrow \mathbb{Z}$ with kernel generated by $\chi$ maps characters $\chi^{\prime}<\chi$ to the negative integers and maps $\chi^{\prime \prime}>\chi$ to the positive integers. These are weights for a $\mathbb{C}^{*}$ action on $\mathbb{C}^{N-k}$. The variation of that action (e.g., as in [Bro99]) describes a (generalised) flip in an affine patch in $\mathcal{Z}_{\alpha}$.

In more complicated cases later, when we consider equations inside the toric flip, we have to shrink this affine neighbourhood to an analytic neighbourhood of the point to describe a flip.

4.1.9. The proof of Theorem 4.1. Given $\mathcal{Y} \longrightarrow \mathcal{X}$, let $\mathcal{N}=C(\mathcal{Y})$ be the Cox ring of $\mathcal{Y}$; by construction, this is a polynomial ring (with $N$ variables, say) with a grading by $\mathbb{Z} \oplus \mathbb{Z}$. Moreover, the left-hand ray $r_{0}$ of $\Sigma_{\text {eff }}$ contains a single character, corresponding to the new element of the 1-skeleton. 
The variation of this quotient described above produces a link diagram as required. (We must omit the quotient by characters in the first chamber $\mathcal{C}_{0}$; it is isomorphic to $\mathcal{X}$, because the left-hand ray $r_{0}$ contains only a single character, and its two morphisms are the structure map from $\mathcal{X}$ to a point and an isomorphism $\mathcal{X} \cong \mathcal{Z}_{1}$. Similarly, we omit the quotient in the final chamber if the previous map is also a divisorial contraction.) The maps are isomorphisms in codimension 1 because the contracted loci, as described by Lemma 4.4, are geometric quotients of (a stable set in) $\mathbb{C}^{k}$ for $k \leq N-2$ by $\mathbb{C}^{*} \times \mathbb{C}^{*}$ and so have dimension at most $N-4$, which is codimension 2 in the quotients. The possible behaviour at the two ends is completely determined by the 2-ray game.

\subsection{Examples}

For calculations, it is convenient to summarise the action by a matrix of integers

$$
\left(\begin{array}{llll}
a_{1} & a_{2} & \cdots & a_{N} \\
b_{1} & b_{2} & \cdots & b_{N}
\end{array}\right)
$$

The lemmas above can then be summarised by a sketch of the layout of this matrix $\chi$ near $\mathcal{Y}_{\alpha}$ :

$$
\left(\begin{array}{c|ccc|c}
I_{\mathcal{F}_{\alpha}} & \multicolumn{3}{|c|}{\mathcal{Y}_{\alpha}} & I_{\mathcal{G}_{\alpha}} \\
& \mathcal{Z}_{\alpha} & \mathcal{Z}_{\alpha+1} & \\
x_{1} \cdots x_{m_{\alpha-1}} & x_{l_{\alpha}} \cdots x_{m_{\alpha}} & x_{l_{\alpha+1}} \cdots x_{m_{\alpha+1}} & x_{l_{\alpha+2}} \cdots x_{N}
\end{array}\right)
$$

where $x_{l_{\alpha}}, \ldots, x_{m_{\alpha}}$ are the generators of $\mathcal{N}$ whose characters lie in the ray $R_{\alpha}$.

4.2.1. Standard flop between two scrolls. Consider the graded polynomial ring

$$
\mathcal{N}=\mathbb{C}\left[x_{1}, x_{2}, y, z_{1}, z_{2}\right] \quad \text { with weights }\left(\begin{array}{lllll}
0 & 0 & 1 & 1 & 1 \\
1 & 1 & 1 & 0 & 0
\end{array}\right)
$$

That is, $x_{1}, \ldots, z_{2}$ are eigencoordinates on $\mathbb{C}^{5}$ for the action of $G=\mathbb{C}^{*} \times \mathbb{C}^{*}$ in which $x_{1}$ is in the $(0,1)$-eigenspace, $y$ is in the $(1,1)$-eigenspace, and so on. Setting

$$
\begin{aligned}
& \mathcal{B}_{0}=\mathbb{C}^{5} \backslash\left\{\left(x_{1}=x_{2}=0\right) \cup\left(y=z_{1}=z_{2}=0\right)\right\}, \\
& \mathcal{A}_{0}=\mathbb{C}^{5} \backslash\left\{\left(x_{1}=x_{2}=y=0\right) \cup\left(y=z_{1}=z_{2}=0\right)\right\}, \\
& \mathcal{B}_{1}=\mathbb{C}^{5} \backslash\left\{\left(x_{1}=x_{2}=y=0\right) \cup\left(z_{1}=z_{2}=0\right)\right\}
\end{aligned}
$$


write

$$
\mathcal{Y}=\mathcal{B}_{0} / G, \quad \mathcal{Z}=\mathcal{A}_{0} / / G, \quad \mathcal{Y}^{\prime}=\mathcal{B}_{1} / G
$$

These three varieties fit in the rank 2 link

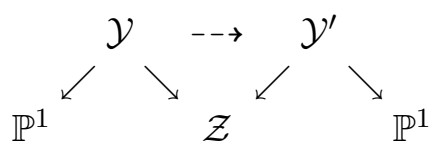

in which the map $\mathcal{Y} \rightarrow-\rightarrow \mathcal{Y}^{\prime}$ is the standard flop. This link is one of the 3 -fold analogues of $\mathbb{P}^{1} \times \mathbb{P}^{1}$ with its two fibration structures.

A different choice of weights such as

$$
\left(\begin{array}{ccccc}
1 & 1 & 1 & 1 & 1 \\
1 & 1 & 0 & -1 & -1
\end{array}\right)
$$

is more symmetric and certainly does determine the same link as that of the original. It has the advantage that one can see immediately that the flip is the $\mathbb{C}^{*}$ flip by quotients of $\mathbb{C}^{4}$ by the $\mathbb{C}^{*}$ action $(1,1,-1,-1)$ - the bottom row of the matrix 'localised' at a point in the base. But the disadvantage is that it presents the flopping variety $\mathcal{Z}^{\prime}$ embedded with quasi reflections for

$$
\mathcal{Z}^{\prime}=\operatorname{Proj} \mathbb{C}\left[y, x_{1} z_{1}, x_{1} z_{2}, x_{2} z_{1}, x_{2} z_{2}\right] \subset \mathbb{P}(1,2,2,2,2) .
$$

4.2.2. Francia antiflip. Consider the action of $G$ on $\mathbb{C}\left[x_{1}, x_{2}, y, z_{1}, z_{2}\right]$ given by weights

$$
\left(\begin{array}{ccccc}
0 & 1 & 1 & 1 & 1 \\
1 & 0 & -1 & -2 & -3
\end{array}\right) .
$$

Calculating the quotients this time describes a Sarkisov link

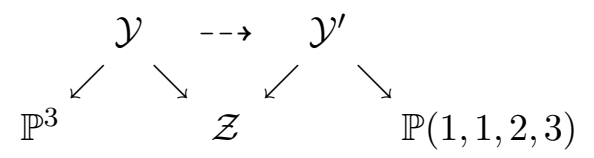

in which one end is the $(1,2,3)$-blowup of a point of $\mathbb{P}^{3}$, the other is the $(1,1,2)$-blowup of a nonsingular point of $\mathbb{P}(1,1,2,3)$, and the map $\mathcal{Y} \rightarrow-\rightarrow \mathcal{Y}^{\prime}$ is the opposite of the Francia flip.

We describe this in terms of toric geometry. Start with the usual fan for $\mathbb{P}^{3}$ in $\mathbb{Z}^{3}$. After introducing the ray $(1,-1,2)$ and subdividing minimally, giving the fan $\Delta \mathcal{y}$, the convex cone

$$
\langle(1,-1,2),(0,1,0),(1,0,0),(0,0,1)\rangle
$$


is divided into two simplexes by the face joining $(1,0,0)$ and $(0,0,1)$. The Francia antiflip is the operation on fans $\Delta \mathcal{Y} \rightsquigarrow \Delta_{\mathcal{Y}^{\prime}}$ that removes this edge and replaces it by the edge joining $(1,-2,1)$ and $(0,1,0)$; indeed, in the notation of [Rei83],

$$
1 \times(1,-1,2)+1 \times(0,1,0)=1 \times(1,0,0)+2 \times(0,0,1) .
$$

Since in $\Delta_{\mathcal{Y}^{\prime}}$

$$
(0,0,1)=1 \times(1,-1,2)+1 \times(-1,-1,-1)+2 \times(0,1,0),
$$

we can remove the ray $(0,0,1)$, the opposite of a $(1,1,2)$-blowup of a nonsingular point. The result is $\mathbb{P}(1,1,2,3)$ because

$$
1 \times(1,0,0)+1 \times(1,-1,2)+2 \times(-1,-1,-1)+3 \times(0,1,0)=(0,0,0) .
$$

As another example, the action

$$
\left(\begin{array}{ccccc}
0 & 1 & 1 & 1 & 1 \\
1 & 0 & -1 & -2 & -5
\end{array}\right)
$$

is similar: the corresponding link starts with the $(1,2,5)$-blowup of $\mathbb{P}^{3}$, makes the antiflip of type $(1,1,-1,-4)$, and finishes with (the inverse of) the Kawamata blowup of $1 / 3(1,1,2)$ in $\mathbb{P}(1,3,4,5)$. One can check that these are the only two such examples of Sarkisov links starting from $\mathbb{P}^{3}$ : more precisely, any other calculation with $N=5$ and ordinary $\mathbb{P}^{3}$ at one end has nonterminal singularities emerging from the antiflip.

4.2.3. Change of structure. The action

$$
\left(\begin{array}{ccccc}
0 & 1 & 1 & 1 & 1 \\
1 & 2 & 0 & -1 & -1
\end{array}\right)
$$

is similar to the first example, being

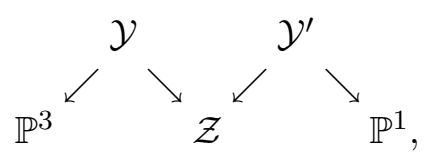

where $\mathcal{Y} \rightarrow \mathcal{Y}^{\prime}$ is the Francia flip - the toric flip with weights $(1,2,-1,-1)$ as in $\left[\right.$ Bro99, Section 1] - and $\mathcal{Y}^{\prime} \rightarrow \mathbb{P}^{1}$ has fibres $\mathbb{P}(1,1,3)$. (In fact, the 3 -folds here each have a line of canonical singularities, so this is not a Sarkisov link: the map $\mathcal{Y} \rightarrow \mathbb{P}^{3}$ is the $(2,3,3)$-blowup of a point, as one sees immediately by localising.)

In the toric fan after this flip, the plane spanned by the new edge comprises cones of the fan, so the variety $\mathcal{Y}^{\prime}$ admits a toric morphism to $\mathbb{P}^{1}$. 


\section{$\S 5$. Embedding 3-fold links in toric links}

\subsection{Finitely generated extremal extractions}

Let $X$ be a Fano 3 -fold, and let $p \in X$ be a point.

Definition 5.1. A morphism $f: Y \rightarrow X$ is an extremal extraction of $p$ if and only if it is an extremal contraction of a divisor $E$ on $Y$ with centre $p \in X$ and there is a member in $\left|-K_{Y}\right|$ that has only Du Val singularities.

We use the notation of [CPR00]: $A=-K_{X}$ and $B=-K_{Y}=f^{*} A-$ $a\left(K_{X}, E\right) E$. The main case we consider is when $p \in X$ is a nontrivial terminal quotient singularity, in which case it is well known that there is exactly one extremal extraction of $p$, the Kawamata blowup (see [Kaw96]).

We define a global version of the algebras considered by Kawakita in [Kaw02].

Definition 5.2. Let $f: Y \rightarrow X$ be an extremal extraction of $p$, and define a $k$-algebra $\mathcal{B}=\bigoplus_{m>0} H^{0}(Y, m B)$ that is graded by $m$. The graded ring of $f$ is the $\mathbb{Z}$-graded $\mathcal{B}$-algebra

$$
\mathcal{M}=\mathcal{M}(f)=\bigoplus_{n \in \mathbb{Z}} \mathcal{M}_{n}
$$

whose $n$th graded piece for each integer $n$ is the graded $\mathcal{B}$-module

$$
\mathcal{M}_{n}=\bigoplus_{m \geq 0} H^{0}(Y, m B-n E)
$$

We consider only cases for which $\mathcal{B}=\bigoplus_{m>0} H^{0}(Y, m B)$ is a finitely generated $k$-algebra graded by $m$. The ring $\mathcal{M}$ is defined as a $\mathcal{B}$-algebra to emphasise its grading by $n$-the order of vanishing of functions on $E$, and ultimately the action responsible for flips in the links - but it is usually regarded as a $k$-algebra having two gradings by $m$ and $n$. In particular, references to generators of $\mathcal{M}$ mean generators as a $k$-algebra unless specified otherwise. The ring structure of $\mathcal{M}$ is determined by multiplication in $k(Y)$. For fixed values of $m, n \in \mathbb{Z}$, there are $k$-subalgebras

$$
R(Y, m B-n E)=\bigoplus_{k \geq 0} H^{0}(Y, k(m B-n E)) \subset \mathcal{M}
$$

and these are zero if $m<0$ or if $m=0$ and $n>0$. We consider such subrings only of $\mathcal{M}$ for $m$ and $n$ coprime. 
Definition 5.3. An extremal extraction $f$ is finitely generated if and only if $\mathcal{M}(f)$ is finitely generated (as a $k$-algebra).

If $f$ is finitely generated and the 2-ray game on $Y$ plays out to a link $\Phi$ from $p \in X$, then loosely speaking we refer to $\mathcal{M}(f)$ as the graded ring of the link $\Phi$. But this is an abuse of terminology: the ring $\mathcal{M}(f)$ exists even if the link does not, and when the link does exist its graded ring, even considered only up to isomorphism, depends on which end is taken to be the start of the link. In any case, $\mathcal{M}(f)$ is of finite index in the Cox ring of $Y$.

We give an elementary proof of the following.

Theorem 5.4. Let $f: Y \rightarrow X$ be a finitely generated extremal extraction. Then the 2-ray game on $Y$ determines a rank 2 link from $X$.

In fact, this follows from [HK00, Proposition 2.9]. Our proof is suited to making calculations in explicit situations, because we do not apply the variation of GIT directly to $Y$, but simply follow the birational transforms of $Y$ through an ambient toric link. The main point is that the link continues to an end and does not fail because some contraction or flip does not exist. We use the ambient toric link to guarantee that such maps exist. A less important point, but one that is interesting from the point of view of calculation, is that the toric link contains only one link from $X$ and not a sequence of links.

Proof. Let $f: X \longrightarrow Y$ be a finitely generated extremal extraction with exceptional divisor $E \subset Y$ from a Fano 3 -fold $X$. Let $\mathcal{M}=\mathcal{M}(f)$ be the graded ring of $f$. By assumption, one can choose generators for $\mathcal{M}$ and write $\mathcal{M}=\mathcal{N} / I$ for a doubly graded polynomial $\operatorname{ring} \mathcal{N}$ and a homogeneous ideal $I \subset \mathcal{N}$.

Claim 5.5. The generators of $\mathcal{M}$ can be chosen so that none lie in $H^{0}(Y, a A+b E)$ with both $a, b>0$.

First, the generating set must include a basis of $H^{0}(Y, E) \cong k$ : for any $c, d>0, H^{0}(Y,-c B+d E)=0($ since $(-c B+d E) C<0$ for all curves $C \subset Y$ that miss $E)$, so $H^{0}(Y, E)$ lies on a boundary edge of the cone $\Sigma_{\text {eff }}$. Now let $H=H^{0}(Y, a A+b E)$ be a graded piece of $\mathcal{M}$ with $a, b>0$. Since $A$ is numerically trivial on $E, a A+b E$ is negative and the cohomology of

$$
0 \rightarrow \mathcal{O}_{Y}(a A+(b-1) E) \rightarrow \mathcal{O}_{Y}(a A+b E) \rightarrow \mathcal{O}_{E}(a A+b E) \rightarrow 0
$$


shows that any element of $H$ is divisible by the generator of $H^{0}(Y, E)$. Thus elements of $H$ are generated by those of $H^{0}(Y, E)$ and $H^{0}(Y, a A+(b-1) E)$, and repeating this process proves the claim.

For such a choice of generators, let

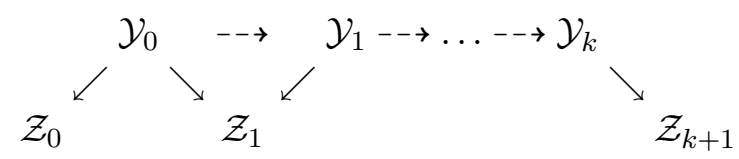

be the toric link arising from the grading on $\mathcal{N}$; assume that the trivial left-hand map (which would come from the basis of $H^{0}(Y, E)$ ) is removed, so that $\mathcal{Z}_{0} \leftarrow \mathcal{Y}_{0}$ is a birational toric blowup. Furthermore, all $\mathcal{Y}_{i}$ have the same dimension, and we may assume that $\mathcal{Y}_{k} \rightarrow \mathcal{Z}_{k+1}$ is either a (birational) divisorial contraction or a map to a toric variety of lower dimension but not a point (again by omitting a trivial right-hand map if necessary).

Claim 5.6. Without loss of generality, $X$ embeds in $\mathcal{Z}_{0}$ and intersects the open toric stratum of $\mathcal{Z}_{0}$ nontrivially.

Certainly $X$ embeds into some quotient of $\mathcal{A}=\operatorname{Spec} \mathcal{N}$, and the choice of generators means that both $X$ and $\mathcal{Z}_{0}$ are the quotients polarised by a character in the first nontrivial ray in $\Sigma_{\text {eff }}$, which is along multiples of $A$ in each case, so $X \hookrightarrow \mathcal{Z}_{0}$.

If the image of this embedding misses the open stratum, then there are generators of $\mathcal{N}$ that map to sections in $\mathcal{M}$ that vanish on $X$. That is, we have chosen a generator $z_{i} \in \mathcal{N}$ that is contained in the ideal $I$. Such generators are redundant and can be omitted (although we must recompute the sequence (5.1)).

Claim 5.7. The map $X \leftarrow Y$ embeds in the map $\mathcal{Z}_{0} \leftarrow \mathcal{Y}_{0}$.

Similarly, using (a suitable multiple of) $A-\varepsilon E$ for small $\varepsilon>0$ shows that $Y \hookrightarrow \mathcal{Y}_{0}$. The maps between quotients are the same since they are defined by (the restriction of) the same linear system. (Many generators may lie between $A$ and $B$ in $\Sigma_{\text {eff }}$, and some choices of polarisation defining $Y$ will embed it naturally in other $\mathcal{Y}_{i}$; nevertheless, characters very close to $A$ polarise the quotients of both $Y$ and $\mathcal{Y}_{0}$.)

Claim 5.8. The birational images $Y_{i} \subset \mathcal{Y}_{i}$ of $Y$ in each $\mathcal{Y}_{i}$ are equal to $\operatorname{Proj} R_{i}$, where $R_{i}=\bigoplus_{j \geq 0} H^{0}\left(Y, j\left(m_{i} B+n_{i} E\right)\right)$ for some integers $m_{i}>0$ and $n_{i} \in \mathbb{Z}$. Moreover, each of these varieties $Y_{i}$ is normal. 
Each $\mathcal{Y}_{i}$ is Proj of some $\mathbb{N}$-graded subring of $\mathcal{N}$ given by the positive multiples of polynomials in $\mathcal{N}$ of bidegree $\left(m_{i}, n_{i}\right)$, for any fixed integer pair $\left(m_{i}, n_{i}\right)$ lying in the chamber of $\Sigma_{\text {eff }}$ corresponding to $\mathcal{Y}_{i}$. The graded subring of $\mathcal{M}$ comprising the summands of these same degrees defines the image $Y_{i} \subset \mathcal{Y}_{i}$ of $Y$. Since $Y$ meets the open torus of $\mathcal{Y}_{0}$, and since all transformations occurring in (5.1) take place on toric strata, each $Y_{i}$ is birational to $Y$. (We also denote $Y_{0}=Y$.) The $Y_{i}$ are normal because they are Proj of the ring of all multiples of an ample divisor; this is Zariski projective normalisation, as in [Rei87], for example.

Similarly, one can define varieties $Z_{i} \subset \mathcal{Z}_{i}$. Again, these are 3 -folds birational to $Y$, with the possible exception of $Z_{k+1}$-in fact, $Z_{k+1}$ is birational to $Y$ if and only if $\mathcal{Y}_{k} \rightarrow \mathcal{Z}_{k+1}$ is birational, again because birationality is detected on the intersection with the open toric stratum. The result is an array of varieties and morphisms similar to (5.1) but composed of $Y_{i}$ and $Z_{i}$. The theorem then follows by showing that this array is a complete 2-ray link from $X$.

Inductively, we assume that $Y_{i}$ is $\mathbb{Q}$-factorial with $\rho_{Y_{i}}=2$; these properties hold for $Y=Y_{0}$.

ClAIM 5.9. If $i<k-1$ and the morphism $Y_{i} \rightarrow Z_{i+1}$ is an isomorphism, then $Z_{i+1} \leftarrow Y_{i+1}$ is also an isomorphism.

Since $Z_{i+1}$ is isomorphic to $Y_{i}, a B-b E$ is an ample $\mathbb{Q}$-Cartier divisor on $Z_{i+1}$ for some $a, b>0$, so the same is true for $a B-(b+\varepsilon) E$ for sufficiently small and rational $\varepsilon>0$. Multiples of this divisor embed $Z_{i+1} \hookrightarrow \mathcal{Y}_{i+1}$. In particular, this map is an isomorphism to its image when restricted to the open toric stratum, so it is the birational map $Z_{i+1} \rightarrow Y_{i+1}$, but this is an isomorphism, which is the claim.

Claim 5.10. Suppose that $Y_{i} \rightarrow Z_{i+1}$ is not an isomorphism and is smallthat is, no divisor is contracted. Then $i<k, Z_{i+1} \leftarrow Y_{i+1}$ is small, and the birational map $Y_{i} \rightarrow Y_{i+1}$ is a flip, flop, or antiflip that corresponds to a step in a 2-ray link.

Let $D=m_{i} B+n_{i} E$ be an ample divisor on $Z_{i+1}$. (This divisor will polarise the resulting flip.) Then on $Y_{i}$, the divisor $D+\varepsilon E$ is positive on the contracted locus for small $\varepsilon>0$ (because it is at the level of toric varieties in $(5.1))$ and

$$
Y_{i} \cong \operatorname{Proj}_{Z_{i+1}} \bigoplus_{n \geq 0} H^{0}\left(Z_{i+1}, n N(D+\varepsilon E)\right)
$$


for suitable fixed (and divisible) $N>0$. Since it is a quotient of some subring of $\mathcal{N}$, the $\mathcal{O}_{Z_{i+1}}$-algebra $\bigoplus_{n \geq 0} H^{0}\left(Z_{i+1}, n N(D-\varepsilon E)\right)$ is finitely generated; its $\operatorname{Proj}_{Z_{i+1}}$ is the required flip, and it embeds the flip in $\mathcal{Y}_{i+1}$. In particular, $Y_{i+1}$ is also $\mathbb{Q}$-factorial with $\rho_{Y_{i+1}}=2$.

Claim 5.11. Suppose that $Y_{i} \rightarrow Z_{i+1}$ is birational and contracts a divisor. Then this map is an extremal divisorial contraction that completes the link and all further maps $Z_{j} \leftarrow Y_{j} \rightarrow Z_{j+1}$ are isomorphisms.

Since $\rho_{Y_{i}}=2$ and since $Z_{i+1}$ is projective, $\rho\left(Z_{i+1}\right)=1$; this contraction is extremal, and $Z_{i+1}$ is also $\mathbb{Q}$-factorial. Thus, if $i<k$, then $Z_{i+1} \leftarrow Y_{i+1}$ must be an isomorphism, as in Claim 5.9 above. Now, inductively for any $j>i, Z_{i+1} \cong Y_{j}$ and $Y_{j} \rightarrow Z_{j+1}$ is an isomorphism (because $Y_{j}$ has $\rho=1$ ); and again, as in Claim 5.9 above, the 'flip' of this is an isomorphism.

Claim 5.12. Suppose that $Y_{i} \rightarrow Z_{i+1}$ is not birational. Then this map is an extremal fibration that completes the link, and $i=k$.

The map is certainly extremal, as usual, since $\rho_{Y_{i}}=2$, so it does complete the link. Since $Y_{i}$ meets the big torus of $\mathcal{Y}_{i}$, this map can fail to be birational only if the toric map $\mathcal{Y}_{i} \rightarrow \mathcal{Z}_{i+1}$ is not birational. This can occur only if $i=k$.

\subsection{Further explicit details of the 3 -fold links}

Here we apply the proof of Theorem 5.4 to analyse further the steps in the birational links of Theorem 1.1. For these links, the finite generation assumption by $\mathrm{Hu}$ and Keel (see [HK00]) holds since the Kawamata blowup of $p \in X$ is a weak Fano 3-fold and so is Mori dream space. But it is interesting that the method of proof of Theorem 5.4 also applies to calculations where finite generation is not yet known. We can construct an algebra $\mathcal{M}^{\prime}$ using bases of linear systems on $X$ that appear naturally (including an ample system, so that $X$ embeds in the resulting toric variety) and then follow $X$ through the toric link associated to a 2-ray game for $\mathcal{M}^{\prime}$. This may break down altogether if we have not selected enough linear systems to start with, but very often either it works or it is clear how to choose a better toric link.

Sections 5.2.1 and 5.2.2 explain the further analysis we do for the links of Theorem 1.1. In particular, these methods compute the flips that occur in the links to del Pezzo fibrations listed in Table 1. The remaining sections sketch calculations for which we do not start out with finite generation but instead compute a link from limited information, correcting it if necessary. 
5.2.1. Calculations in the ambient toric 2-ray link. The key point in all of the cases here is that by Lemma 3.6 the generators of the unprojection ideal all vanish to order 1 along the unprojection divisor. This is a consequence not of the Type I definition but of the form of the Pfaffian equations. (The same holds for Type I in codimension $\leq 3$. However, this cannot be expected in higher codimension; in codimension 4 , [BKR] includes examples with vanishing of order 2.)

We generate a subring of the graded ring of the link. (We use notation here that is convenient for this calculation only, and it should not be confused with that of Notation 3.3.) Recall that the link starts with the Kawamata blowup of a terminal quotient singularity $p \in X$ of type $(1 / r)(1, a, r-a)$ followed by a flopping contraction: $X \leftarrow Y \rightarrow Z \supset E$, where $E \subset Z$ is the image of the exceptional divisor of the blowup. Let $I_{E \subset Z}=\left(y_{1}, y_{2}, y_{3}\right)$ and $x_{1}, x_{2}, x_{3}$ be coordinates on $E \cong \mathbb{P}(1, a, r-a)$. The weights $a_{i}, b_{j}$ of the variables $y_{i}, x_{j}$ translate on $Y$ as

$$
x_{i} \in H^{0}\left(Y, b_{i} B\right), \quad y_{i} \in H^{0}\left(Y, a_{i} B-E\right),
$$

where $a_{1} \leq a_{2} \leq a_{3}$, as usual. Including a generating section $e \in H^{0}(Y, E)$ and the projection variable $z \in H^{0}(Y, c B+E)$, we have the subring

$$
\mathcal{M}^{\prime}:=\mathbb{C}\left[e, z, x_{1}, x_{2}, x_{3}, y_{3}, y_{2}, y_{1}\right] \subset \mathcal{M}
$$

Of course, the given generators of $\mathcal{M}^{\prime}$ are subject to relations. Writing $\mathcal{M}^{\prime}=\mathcal{N} / I$ for a polynomial ring $\mathcal{N}$ with generators in the same bidegrees as those of $\mathcal{M}^{\prime}$, we recover a toric link. We do not concern ourselves with whether $\mathcal{M}^{\prime}=\mathcal{M}$ because, in the cases we consider, the toric link is enough to describe the Sarkisov link from $X$.

FAmily No. 10. Here $\left(X \subset \mathbb{P}^{6}(1,1,1,2,2,2,3)\right.$ with degree $=13 / 6$ and basket $=\{3 \times 1 / 2(1,1,1), 1 / 3(1,1,2)\})$. We describe $\mathcal{M}^{\prime}=\mathcal{N} / I$ in this case together with the action of $\mathbb{C}^{*} \times \mathbb{C}^{*}$ given by a matrix of weights. The link that starts by projecting from the $1 / 3(1,1,2)$ singularity determines the (ordered) character matrix

$$
\chi=\left(\begin{array}{cccccccc}
0 & 3 & 1 & 1 & 2 & 2 & 2 & 1 \\
-1 & -1 & 0 & 0 & 0 & 1 & 1 & 1
\end{array}\right)
$$

which determines an action of $G=\mathbb{C}^{*} \times \mathbb{C}^{*}$ on $\mathbb{C}^{8}$. The coordinates have bidegrees given by the columns of the matrix, and we name them as $e, z, x_{1}$, 
$x_{2}, x_{3}, y_{1}, y_{2}, y_{3}$, in that order. The ideal $I$ is generated by the maximal Pfaffians of the skew $5 \times 5$ matrix

$$
\left(\begin{array}{cccc}
z & A_{3,0} & B_{2,0} & C_{2,0} \\
& D_{3,0} & E_{2,0} & F_{2,0} \\
& & y_{1} & -y_{2} \\
& & & y_{3}
\end{array}\right),
$$

where $A=A\left(x_{1}, x_{2}, x_{3}, e y_{1}, e y_{2}, e y_{3}\right)$ has bidegree $(3,0)$, and analogous statements for $B, \ldots, F$. Let $\mathcal{A}=V(I) \subset \mathbb{C}^{8}$.

The quotient $\mathcal{A} /{ }_{(3,-1)} G$ gives $X$, although not in the embedding in $\mathbb{P}^{6}$ above since that is polarised by $A=B+1 / 3 E$, which is not an element of $\mathcal{N} / I$. It is easier to see that the quotient $\mathcal{A} /{ }_{(1,0)} G$ is the projection of $X$ from its $1 / 3$ point: each term $z y_{i}$ is eliminated by one of the Pfaffian equations.

We could compute a graded ring on $X^{\prime}$, the end of the link, but again it would appear in some Veronese embedding. The unprojection calculation below is a better method for computing $X^{\prime}$.

The link that starts by projecting from one of the $1 / 2(1,1,1)$ singularities determines the (ordered) character matrix

$$
\chi=\left(\begin{array}{cccccccc}
0 & 2 & 1 & 1 & 1 & 3 & 2 & 2 \\
-1 & -1 & 0 & 0 & 0 & 1 & 1 & 1
\end{array}\right)
$$

A row operation on the character matrix renders it as

$$
\left(\begin{array}{cccccccc}
3 & 5 & 1 & 1 & 1 & 0 & -1 & -1 \\
-1 & -1 & 0 & 0 & 0 & 1 & 1 & 1
\end{array}\right)
$$

and equations for $y_{1} z$ and $y_{1} x_{i}$ for some $i$ eliminate two of the variables in a neighbourhood of $P_{y_{1}}$, so after the usual flop we see a toric hypersurface flip of type $(3,1,1,-1,-1 ; 2)$, where the 2 indicates the weight of the hypersurface. The resulting 3 -fold has a fibration to $\mathbb{P}^{1}$, given by the ratio of the last two variables, with cubic surfaces as the fibres - these can already be seen in the syzygy matrix.

The flip occurring in the example above was one of the hypersurface flips of [Bro99, Theorem 8.1]. In fact, every flip that appears in Theorem 1.1 is either a toric flip or a hypersurface flip. This seems to be simply an artifact of working in fairly low codimension. The proof of Theorem 1.1 shows that the subalgebra $\mathcal{M}^{\prime}$ that we work with is sufficient to describe the link on $X$, 
so the flip is at most codimension 3. Using the notation of Proposition 3.2, the fact that the variable $t$ appears linearly in the syzygy matrix of $X$ means that there is at least one linear equation in a neighbourhood of the flipping locus, and this reduces the codimension of the flip to at most 2. Finally, a case-by-case check on the degrees of the forms $C$ and $F$ in the same syzygy matrix confirms that there is at least one further independent linear equation in a neighbourhood of the flipping locus when these forms are chosen to be general. All the same, it is amazing how effective the ambient toric links are for describing the various generalised flips that occur in these links.

5.2.2. Unprojection and the equations at the end of the link. When the link ends with another Fano 3-fold, we can compute its equations by adapting Reid and Takagi's example of a so-called Type III unprojection (see [Rei00, Example 9.16]). We sketch the general approach and then give a typical example of the method.

Consider $\mathbb{C}^{10}$ with coordinates $t, x, x_{1}, x_{2}, A, B, C, D, E, F$. Let $V \subset \mathbb{C}^{10}$ be the 7 -fold defined by the five maximal Pfaffians of the following $5 \times 5$ skew matrix (where, as usual, we omit the diagonal of zeros and the skew lower triangular half):

$$
\left(\begin{array}{cccc}
t & A & B & C \\
& D & E & F \\
& & x_{2} & x_{1} \\
& & & x
\end{array}\right) .
$$

Of course, $V \cong \mathcal{C} \operatorname{Grass}(2,5)$, the cone on the Grassmannian in its Plücker embedding, and the equations are neatly expressed in terms of matrix entries $m_{i j}$ by

$$
\varphi_{\ell}=m_{h i} m_{j k}-m_{h j} m_{i k}+m_{h k} m_{i j}
$$

for the five ways of choosing $\{h, i, j, k, \ell\}=\{1,2,3,4,5\}$ with $h, i, j, k$ in increasing order.

The elimination of $t$ (which appears only in Pfaffians $\varphi_{3}, \varphi_{4}, \varphi_{5}$ ) from these equations determines a birational map from $V$ to

$$
W:\left(\varphi_{1}=\varphi_{2}=0\right) \subset \mathbb{C}^{9}
$$


The locus $(x=0)$ in $W$ splits into two components $E \cup F$, where the ideals of these loci in $\mathbb{C}^{9}$ are

$$
I_{E}=\left(x, x_{1}, x_{2}\right) \quad \text { and } \quad I_{F}=\left(x, \bigwedge\left(\begin{array}{cc}
B & C \\
E & F \\
x_{2} & -x_{1}
\end{array}\right)\right)
$$

and $\wedge^{2} M$ denotes the three $2 \times 2$ minors of a matrix $M$. In fact, $E$ is the unique exceptional divisor of $W \rightarrow V$, and the unprojection construction we describe next produces another birational map $W \rightarrow U-$ the so-called Type III unprojection - for which $F$ is the unique exceptional divisor.

Unprojections can be computed by comparing free resolutions, so setting $\mathcal{O}=\mathcal{O}_{\mathbb{C}^{9}}$, we write

$$
\begin{array}{lllll}
0 \leftarrow \mathcal{O}_{F} \longleftarrow \mathcal{O} & \stackrel{M_{0}}{\longleftarrow} & 4 \mathcal{O} & \stackrel{M_{1}}{\longleftarrow} & 5 \mathcal{O} \stackrel{M_{2}}{\longleftarrow} 2 \mathcal{O} \leftarrow 0 \\
\uparrow & J_{1} \uparrow & J_{2} \uparrow \\
0 \leftarrow \mathcal{O}_{X} \longleftarrow \mathcal{O} & \stackrel{\left(\varphi_{2},-\varphi_{1}\right)}{\longleftarrow} 2 \mathcal{O} \stackrel{t\left(\varphi_{1}, \varphi_{2}\right)}{\longleftarrow} \mathcal{O} \leftarrow 0
\end{array}
$$

where the free resolution of $\mathcal{O}_{F}$ is computed by $M_{0}=\left(-x, \varphi_{1}, \varphi_{2}, \varphi_{3}\right)$ and

$$
M_{1}=\left(\begin{array}{ccccc}
\varphi_{1} & \varphi_{2} & \varphi_{3} & \varphi_{4} & \varphi_{5} \\
x & 0 & 0 & -C & B \\
0 & x & 0 & -F & E \\
0 & 0 & x & -x_{1} & x_{2}
\end{array}\right), \quad M_{2}=\left(\begin{array}{cc}
B & C \\
E & F \\
x_{2} & x_{1} \\
0 & x \\
-x & 0
\end{array}\right)
$$

and the comparison maps are

$$
J_{1}=\left(\begin{array}{cc}
0 & 0 \\
1 & 0 \\
0 & 1 \\
0 & 0
\end{array}\right), \quad J_{2}=\left(\begin{array}{c}
A \\
D \\
0 \\
-x_{2} \\
-x_{1}
\end{array}\right)
$$

The unprojection is then the solution of the equation

$$
J_{2}(-1)=M_{2}\left(\begin{array}{l}
s_{1} \\
s_{2}
\end{array}\right)
$$

where $s_{1}, s_{2}$ are the standard basis of $2 \mathcal{O}$, the domain of $M_{2}$, and -1 is the basis of $\mathcal{O}$, the domain of $J_{2}$. (Note that $s_{1}, s_{2}$ both have a simple pole along 
the divisor $F$, as usual for unprojection variables.) That is, we consider $\mathbb{C}^{11}$ with new variables $s_{1}, s_{2}$ and define $U \subset \mathbb{C}^{11}$ by the five equations (5.2). Of course, two of the equations are $x_{1}=x s_{1}$ and $x_{2}=x s_{2}$, so one would usually eliminate these two variables to get

$$
U:\left(A+B s_{1}+C s_{2}=D+E s_{1}+F s_{2}=0\right) \subset \mathbb{C}^{9} .
$$

Two twists make this applicable for us. First, the whole construction can be graded with weights chosen on the variables for which the Pfaffians are homogeneous. In that case, the resolutions are also graded, and one can take Proj of the rings appearing to give birational maps between varieties in WPS. Second, the variables can be specialised to give calculations in lower dimensions; typical for us is to set $A, \ldots, F$ to be functions of three new variables together with the $x$ variables so that we work with projective 3-folds.

Family No. 9. Here $\left(X \subset \mathbb{P}^{6}(1,1,1,3,4,5,6)\right.$ with degree $=5 / 6$ and basket $=\{1 / 6(1,1,5)\})$. The link from the $1 / 6(1,1,5)$ point will be a Kawamata blowup, a flop, and a hypersurface flip of type $(4,1,1,-3,-1 ; 2)$ followed by the contraction of a divisor to a point on a Fano 3 -fold $X^{\prime}$. The unprojection method computes the equations of $X^{\prime}$ from the syzygy matrix of $X$. In variables $x, x_{1}, x_{2}, y, z, t, u$, this matrix is

$$
\left(\begin{array}{cccc}
u & A_{7} & B_{5} & C_{4} \\
& t & E_{3} & F_{2} \\
& & z & y \\
& & & x
\end{array}\right),
$$

where $A, \ldots, F$ are general forms of the indicated degrees.

The projection $X \rightarrow Z$ from $P_{u}$ works by eliminating $u$ as usual. To continue the link, we introduce two new variables, $s_{1}$ and $s_{2}$ of weights 2 and 3 , respectively, subject to relations

$$
\left(\begin{array}{ccc}
A_{7} & B_{5} & C_{4} \\
t & E_{3} & F_{2} \\
0 & z & y \\
-z & 0 & x \\
-y & -x & 0
\end{array}\right)\left(\begin{array}{c}
1 \\
s_{1} \\
s_{2}
\end{array}\right)=0
$$

where the big matrix is the final three columns of the syzygy matrix. We use these equations to eliminate $y, z$, and also $t$, so the resulting variety is

$$
X^{\prime}:\left(A_{7}+s_{1} B_{5}+s_{2} C_{4}=0\right) \subset \mathbb{P}^{4}(1,1,1,2,3)
$$


given by an equation of degree 7 in variables $x, x_{1}, x_{2}, s_{1}, s_{2}$ (after substituting for $y, z, t)$.

Notice that $X^{\prime}$ contains the $s_{1} s_{2}$ line $\mathbb{P}(2,3)$ and that for general $A$ it has a single node in the interior of this line - this point is the image of the contracted divisor. Thus $X^{\prime}$ is very far from being a general element of the family of all such hypersurfaces. This is typical and already observed in [CM04]. Another point is that the model case of Type III unprojection in [Rei00] is a (rational) divisorial contraction to a line, so this example exhibits something new.

5.2.3. Missing generators. The calculations above apply to many other situations. It can happen that one does not compute the full graded ring $\mathcal{M}$ at first attempt. For example, in the del Pezzo fibrations of Brown, Corti, and Zucconi (see [BCZ04]), birational links are started without enough generators of $\mathcal{M}(f)$ to realise them. The typical indication of missing generators is when a flipping contraction of $Y_{i}$ is not completed to a flip by the ambient toric flip - rather than extracting a new curve on $Y_{i+1}$, the toric transformation may extract a surface (leaving $Y_{i+1}$ non- $\mathbb{Q}$-factorial). Unprojection is the key to identifying the missing generator. This is explained in [BCZ04, Section 4.4.3].

5.2.4. Elliptic and K3 fibrations of Ryder. Ryder [Ryd02] (and similar results in [Ryd06]) shows that the general Fano 3 -fold $X=X_{22} \subset \mathbb{P}(1,1,3$, 7,11 ) is birational to a variety fibred over $\mathbb{P}^{1}$ in $K 3$ surfaces by computing a 2-ray link. Although we do not know finite generation automatically in this case (since $B=-K_{Y}$ is not nef on the Kawamata blowup $Y$ of $X$ ), we can still apply the proof of Theorem 5.4, working on a rank 2 toric variety that we guess by knowing some linear systems on $X$; again, we make no attempt to compute the full ring $\mathcal{M}(f)$ for the Kawamata blowup $f$ but compute just enough of it to see the link.

Given the matrix of weights of a $G=\mathbb{C}^{*} \times \mathbb{C}^{*}$ action on $\mathbb{C}^{6}$,

$$
\left(\begin{array}{cccccc}
0 & 3 & 11 & 7 & 1 & 1 \\
1 & 1 & 3 & 1 & 0 & 0
\end{array}\right)
$$

consider the general hypersurface of bidegree $(22,6)$. One can check that the quotient linearised by $\chi=(3,1)$ is the Fano $X$. The link proceeds by blowing up the $1 / 3(1,1,2)$ quotient singularity $X \leftarrow Y$. The first toric modification (a toric flip with base the quotient $\mathbb{C}^{6} / G$ linearised by $(11,3)$ ) happens away from $Y$, because if $z$ is the variable of weight $(11,3)$, the 
equation of $Y$ includes a $z^{2}$ term. But the second toric modification induces an antiflip of hypersurface type $(-7,-4,-10,1,1 ;-20)$. Writing down equations shows that the flipping curve passes through the two singularities $1 / 7(1,3,4), 1 / 2(1,1,1) \in Y$. The antiflip $Y \rightarrow Y^{\prime}$ results in a nonsingular variety that has a morphism to $\mathbb{P}^{1}$. Again, the equations show that the fibre is a hypersurface of degree 6 in $\mathbb{P}(1,1,1,3)$; the general fibre is nonsingular and so is a K3 surface.

The weight matrix used here could be deduced using calculations similar to [CPR00, Section 4.10], computing the vanishing of the coordinates on $\mathbb{P}(1,1,3,7,11)$ on the exceptional divisor of $X \leftarrow Y$. However, in this case it is likely that we have not calculated the full graded ring $\mathcal{M}$, since presumably other generators are coming from the linear systems of $10 B+$ $2 E, 13 B+3 E, 16 B+4 E, 19 B+5 E$. But we already have enough sections to compute the link, so we do not look for these extra generators.

\section{REFERENCES}

[ABR02] S. Altınok, G. Brown, and M. Reid, "Fano 3-folds, $K 3$ surfaces and graded rings" in Topology and Geometry: Commemorating SISTAG, Contemp. Math. 314, Amer. Math. Soc., Providence, 2002, 25-53.

[AKMW02] D. Abramovich, K. Karu, K. Matsuki, and J. Włodarczyk, Torification and factorization of birational maps, J. Amer. Math. Soc., 15 (2002), 531-572.

[Alt98] S. Altınok, Graded rings corresponding to polarised $K 3$ surfaces and $\mathbb{Q}$-Fano 3-folds, Ph.D. dissertation, University of Warwick, Coventry, 1998.

[BCZ04] G. Brown, A. Corti, and F. Zucconi, "Birational geometry of 3-fold Mori fibre spaces" in The Fano Conference, Univ. Torino, Turin, 2004, 235-275.

[BDK+] G. Brown, S. Davis, A. Kasprzyk, M. Kerber, O. Sisask, and S. Tawn, The graded ring database webpage, grdb.lboro.ac.uk, 2004-2010.

[BKR] G. Brown, M. Kerber, and M. Reid, K3 surfaces and Fano 3-folds, Tom and Jerry, in preparation.

[Bro99] G. Brown, Flips arising as quotients of hypersurfaces, Math. Proc. Cambridge Philos. Soc., 127 (1999), 13-31.

[Bro07] G. Brown, A database of polarized K3 surfaces, Experiment. Math., 16 (2007), 7-20.

[CM04] A. Corti and M. Mella, Birational geometry of terminal quartic 3-folds, I, Amer. J. Math., 126 (2004), 739-761.

[Cor96] A. Corti, Del Pezzo surfaces over Dedekind schemes, Ann. of Math. (2), 144 (1996), 641-683.

[Cor00] A. Corti, "Singularities of linear systems and 3-fold birational geometry" in Explicit Birational Geometry of 3-Folds, London Math. Soc. Lecture Note Ser. 281, Cambridge Univ. Press, Cambridge, 2000, 259-312.

[Cox95] D. A. Cox, The homogeneous coordinate ring of a toric variety, J. Algebraic Geom., 4 (1995), 17-50. 
[CPR00] A. Corti, A. Pukhlikov, and M. Reid, "Fano 3-fold hypersurfaces" in Explicit Birational Geometry of 3-Folds, London Math. Soc. Lecture Note Ser. 281, Cambridge Univ. Press, Cambridge, 2000, 175-258.

[CR00] A. Corti and M. Reid, eds., Explicit Birational Geometry of 3-Folds, London Math. Soc. Lecture Note Ser. 281, Cambridge Univ. Press, Cambridge, 2000.

[CR02] A. Corti and M. Reid, "Weighted Grassmannians" in Algebraic Geometry, de Gruyter, Berlin, 2002, 141-163.

[CS05] A. Corti and I. Smith, Conifold transitions and Mori theory, Math. Res. Lett., 12 (2005), 767-778.

[DH98] I. V. Dolgachev and Y. Hu, Variation of geometric invariant theory quotients, Publ. Math. Inst. Hautes Études Sci. (1998), no. 87, 5-56.

[Dol03] I. Dolgachev, Lectures on Invariant Theory, London Math. Soc. Lecture Note Ser. 296, Cambridge Univ. Press, Cambridge, 2003.

[FS04] O. Fujino and H. Sato, Introduction to the toric Mori theory, Michigan Math. J., 52 (2004), 649-665.

[Gri01] M. M. Grinenko, On fibrations into del Pezzo surfaces, Mat. Zametki, 69 (2001), 550-565.

[HK00] Y. Hu and S. Keel, Mori dream spaces and GIT, Michigan Math. J., 48 (2000), 331-348.

[IF00] A. R. Iano-Fletcher, "Working with weighted complete intersections" in Explicit Birational Geometry of 3-Folds, London Math. Soc. Lecture Note Ser. 281, Cambridge Univ. Press, Cambridge, 2000, 101-173.

[Kaw88] Y. Kawamata, Crepant blowing-up of 3-dimensional canonical singularities and its application to degenerations of surfaces, Ann. of Math. (2), 127 (1988), 93-163.

[Kaw96] Y. Kawamata, "Divisorial contractions to 3-dimensional terminal quotient singularities" in Higher-Dimensional Complex Varieties (Trento, Italy, 1994), de Gruyter, Berlin, 1996, 241-246.

[Kaw02] M. Kawakita, Divisorial contractions in dimension three which contract divisors to compound $A_{1}$ points, Compos. Math., 133 (2002), 95-116.

[Kaw05] M. Kawakita, Three-fold divisorial contractions to singularities of higher indices, Duke Math. J., 130 (2005), 57-126.

[KM98] J. Kollár and S. Mori, Birational Geometry of Algebraic Varieties, Cambridge Tracts in Math., 134, Cambridge Univ. Press, Cambridge, 1998.

[Kol92] J. Kollár, ed., Flips and Abundance for Algebraic Threefolds, Soc. Math. France, Paris, 1992.

[Kol97] J. Kollár, Polynomials with integral coefficients, equivalent to a given polynomial, Electron. Res. Announc. Math. Soc., 3 (1997), 17-27.

[MM82] S. Mori and S. Mukai, Classification of Fano 3-folds with $B_{2} \geq 2$, Manuscripta Math., 36 (1981/1982), 147-162.

[Pap06] S. A. Papadakis, Type II unprojection, J. Algebraic Geom., 15 (2006), 399414.

[Rei83] M. Reid, "Decomposition of toric morphisms" in Arithmetic and Geometry, Vol. II, Progr. Math., 36, Birkhäuser Boston, Boston, 1983, 395-418.

[Rei87] M. Reid, "Young person's guide to canonical singularities" in Algebraic Geometry, Bowdoin, 1985 (Brunswick, Maine, 1985), Proc. Sympos. Pure Math., 46, Amer. Math. Soc., Providence, 1987, 345-414.

[Rei92] M. Reid, What is a flip? unpublished manuscript of Utah seminar, 1992. 
[Rei97] M. Reid, "Chapters on algebraic surfaces" in Complex Algebraic Geometry (Park City, Utah, 1993), IAS/Park City Math. Ser., 3, Amer. Math. Soc., Providence, 1997, 3-159.

[Rei00] M. Reid, "Graded rings and birational geometry" in Proceedings of Algebraic Geometry Symposium (Kinosaki), 2000, 1-72.

[Rei02] M. Reid, Quasi-Gorenstein unprojection, unpublished manuscript, 2002.

[Ryd02] D. Ryder, Elliptic and K3 fibrations birational to Fano 3-fold weighted hypersurfaces, Ph.D. dissertation, University of Warwick, Coventry, 2002.

[Ryd06] D. Ryder, Classification of elliptic and $K 3$ fibrations birational to some $\mathbb{Q}$ Fano 3-folds, J. Math. Sci. Univ. Tokyo, 13 (2006), 13-42.

[Tak06] H. Takagi, Classification of primary $\mathbb{Q}$-Fano threefolds with anti-canonical Du Val K 3 surfaces, I, J. Algebraic Geom., 15 (2006), 31-85.

[Tha96] M. Thaddeus, Geometric invariant theory and flips, J. Amer. Math. Soc., 9 (1996), 691-723.

Gavin Brown

School of Mathematics

Loughborough University

Leicestershire LE11 3TU

United Kingdom

G.D.Brown@lboro.ac.uk

Francesco Zucconi

Dipartimento di Informatica e Matematica

Università di Udine

33100 Udine

Italy

zucconi@dimi.uniud.it 